\title{
Materials informatics with PoreBlazer v4.0 and CSD MOF database
}

\author{
Lev Sarkisov ${ }^{1 *}$, Rocio Bueno-Perez ${ }^{2}$, Mythili Sutharson ${ }^{2}$, and David Fairen-Jimenez ${ }^{2}$ \\ ${ }^{1}$ The Department of Chemical Engineering and Analytical Science / Office C56 The Mill / The \\ University of Manchester / Sackville Street, Manchester, M13 9PL, UK
}

2The Adsorption \& Advanced Materials Laboratory ( $\left.A^{2} M L\right)$, Department of Chemical Engineering \& Biotechnology, University of Cambridge, Philippa Fawcett Drive, Cambridge, CB3 OAS, UK

\begin{abstract}
The development of computational methods to explore crystalline materials has received significant attention in the last decades. Different codes have been reported to help researchers to evaluate and learn about the structure of materials and to understand and predict their properties. Here, we present an updated version of PoreBlazer, an open-access, open-source Fortran 90 code to calculate structural properties of porous materials. The article describes the properties calculated by the code, their physical meaning and their relationship to the properties that can be measured experimentally. Here, we reflect on the methods in the code and discuss features of the most recent version. First, we demonstarte the capabilities of PoreBlazer on the prototypical metal-organic framework (MOF) materials, HKUST-1, IRMOF-1 and ZIF-8, and compare the results to those obtained with other codes, Zeo++ and RASPA. Second, we apply PoreBlazer to the recently assembled database of MOF materials - the CSD MOF subset - and compare properties such as accessible surface area and pore volume from PoreBlazer and the two other codes, and reflect on the possible sources of the differences. Finally, we use PoreBlazer to illustrate how correlations between various structural characteristics can be mined using interactive, dynamic data visualization and how material informatics approaches including principal component analysis and machine learning - can accelerate the discovery of new materials and new functionalities. The results of these calculations, along with the PoreBlazer code, documentation, and case studies are available online from https://github.com/SarkisovGroup/PoreBlazer. The data visualization tool is available at https://aamlexplorer-geo-prop.herokuapp.com), and the principal component analysis is available at https://aamlpca-geo-prop.herokuapp.com.
\end{abstract}

\section{Introduction}

Structure determines property. This simple and powerful concept in chemistry has been the cornerstone of modern computational approaches to drug discovery, where millions of candidate small organic molecules are screened based on their ability to bind to the therapeutic target. Organic chemistry provides the building blocks for metal-organic frameworks (MOFs) ${ }^{1}$. Combined with the large number of topologies into which these building blocks can be arranged, this implies a virtually infinite number of possible MOF structures. As a result, computational screening has become a new, essential tool for porous, crystalline material discovery and optimization, and in particular, for MOFs, zeolitic imidazolate frameworks (ZIFs) ${ }^{2}$, covalent organic frameworks $(\mathrm{COFs})^{3}$, and other classes of materials.

The first example of a virtual MOF designed for a specific application was provided by Duren et al. ${ }^{4}$ They explored how adsorption of methane at 35 bar and room temperature (conditions relevant for 
the adsorbed natural gas vehicle technology) depended on the properties of porous materials, such as the specific surface area and MOF-methane interaction strength. Using insights obtained from computer simulations, the authors proposed new, hypothetical MOFs, with enhanced methane storage capabilities. In the study, the authors also posed a question on how various structural characteristics of a MOF, such as surface area and pore volume, define their functionality in a particular application. Other characteristics of the porous morphology of a MOF include the shape and size of the pores, shape and size of the windows between the pores, access to specific active sites, and so on. Some of these properties have also a particular importance as they can be measured experimentally (e.g. surface area, pore volume, and window size). Collectively, these characteristics the textural properties of materials - form a geometric identity of a MOF, its unique fingerprint.

The tinker-toy nature of MOFs, i.e. the fact that they are assembled from basic building blocks, led to a new, profound idea: we can build databases of virtual MOF materials, following some assembly algorithms, and explore their properties in silico with a view of identifying the most promising candidates for a particular application. These ideas were put together by Wilmer et al. ${ }^{5}$, who constructed a database of 137,953 hypothetical MOFs and investigated their capability to store methane at the target conditions ( 35 bar, room temperature) as a function of their surface area, void fraction, largest pore diameter, etc. Later, this led to a burgeoning area of computational material screening in application to a number of problems, from carbon capture to toxic gas and warfare chemical detection ${ }^{6}$.

For systematic comparison of the materials in computational screening, for their classification and to reveal structure-property relations it is important to have computational tools, which would produce the geometric identity of a MOF. The development of these tools to obtain structural characteristics of porous materials has been a result of many contributions scattered over the years. For example, the algorithms adopted by Duren et $a l^{4,7}$, have been originally applied to characterize molecular models of porous glasses by Gelb and Gubbins ${ }^{8-10}$, which in their turn originate from the methods developed in the field of stereology. Application of Voronoi tessellation in the context of random heterogeneous media can be tracked down to the early eighties ${ }^{11}$. In the last 10 years, several software packages and web platforms have emerged that, given coordinates of the atoms or particles constituting the material, produce a comprehensive set of geometric characteristics. Let us briefly review these codes here before we formulate the objectives of the article.

Chronologically, PoreBlazer, developed by Sarkisov, was the first simulation package of this kind for computational characterization of crystalline and amorphous materials ${ }^{12,13}$. The code, written in Fortran 90, is based on the grid (or lattice) representation of the porous space and calculates pore volume, accessible surface area, largest pore diameter, pore limiting diameter, pore size distribution and other properties. ZEOMICS and MOFomics, by Floudas and co-workers, represent an alternative approach. ${ }^{14,15}$ There, the porous space of the material is parsed into geometric objects (portals, channels, cages) using Delaunay triangulation and complementary geometric methods. Then, the connectivity between these objects is determined and the properties of the structure (accessible surface area, accessible volume) are calculated. These tools are presented in the form of a web portal, where users can submit their structure files and receive the final results by email. Zeo++, developed by Haranczyk and co-workers, is a $\mathrm{C}++$ package for high-throughput analysis of porous materials based on Voronoi tessellation ${ }^{16-18}$. With Voronoi network being a dual graph of the Delaunay network, this approach is closely related to that of Foster et al. ${ }^{19}$. The program is downloadable from the website of 
the developers, with the source code available upon request. RASPA simulation package, developed by Dubbeldam and co-workers, is a powerful, open-source suite of classical simulation tools (e.g. molecular dynamics, Monte Carlo simulations) ${ }^{20}$. Within RASPA, there are options to obtain the surface area and pore volume of the material, using the computational helium porosimetry approach, as well as a pore size distribution.

These software programs differ in their methodology, accessibility, operation, and performance, and we encourage the readers to use a program suited for their specific research needs. However, we believe what is important to do, is to compare properties calculated by these packages to each other. This would ensure consistency across various calculations and methods and would allow us to reflect on the differences in the obtained values as a result of different algorithms and definitions employed. This is prompted by the aspirations of the computational scientific community to improve the consistency of the simulations and reproducibility of the published results ${ }^{21}$. Hence, the objectives of the article can be formulated as follows:

1) To provide a review of the most recent version of PoreBlazer (from now on, PB v4.0), including setup examples, input files, properties it calculates and the algorithms behind these calculations.

This objective is dealt with in Sections 2 on the Properties and Algorithms. We also use this section as an opportunity to establish a clear link between the geometric properties and the actual properties of materials that can be directly or indirectly inferred from the experiments.

2) To establish consistency of the properties calculated across various codes, $P B$ v4.0, Zeo++, and RASPA*

For this, we first consider three specific cases of well-known MOFs (HKUST-1, IRMOF-1 and ZIF-8) to provide a detailed comparison of the properties produced by different codes. We guide the reader on how to set up PB v4.0 simulations and interpret them and also provide the reader with the complete setups used to obtain this data in PB v4.0, Zeo++ and RASPA. We then focus on the recently developed database of MOF structures, the Cambridge Structural Database (CSD) MOF subset. To assemble this database, Moghadam et al. sieved through the CSD to identify entries that satisfy certain criteria characteristic for $\mathrm{MOFs}^{22}$. Using this set of MOFs (see additional details below in Section 3), we apply PB v4.0, Zeo++ and RASPA to obtain key structural characteristics, compare the data produced by the codes and provide an interpretation of the trends. The complete database of the geometric properties obtained for CDS MOF using PB v4.0 is also available for download from https://github.com/SarkisovGroup/PoreBlazer.

3) Provide the reader with a case study of how geometric characterization tools and data can be used in the context of material informatics.

The data produced by PoreBlazer for CSD MOF subset structures form a multidimensional space of values, with the dimensions being the geometric properties. Although interesting functional and topological relations may exist between structures, they are not often easy to reveal and visualize due to the complexity of the space. The set of python codes developed in the Fairen-Jimenez group provides an interactive way to visualize these properties and relations between them ${ }^{23-25}$. In the final

*Unfortunately, at the time of writing this article, the MOFomics and ZEOMICs platforms were not available for use. 
part of this article, we consider how the application of these visualization tools can reveal trends, which help to accelerate the discovery and design of materials with specific adsorptive behavior.

\section{Properties and algorithms}

In this section, we define the geometric properties of interest and their connection to experimentally measured characteristics. We then provide additional details on the algorithms to calculate these properties and how these algorithms have been implemented in PB v4.0.

\subsection{Surface area and pore volume}

Consider the schematic illustration in Figure 1A. The system consists of a porous material, shown as the grey area, and a channel spanning the system from left to right. Atoms of the structure forming the channel are shown as striped circles. Consider now a probe particle of zero size (a point) moving on the surface of the atoms of the structure. In a three-dimensional system, this will create the socalled van der Waals surface, delineating the boundary of the spherical atoms. In the two-dimensional schematic we use, this property is represented by the red line (Figure 1B). The volume enclosed by this surface is called the geometric pore volume, $V_{G}$, which is the volume accessible to a point probe, and it is shown as the light red shaded area in Figure $1 \mathrm{~B}$.

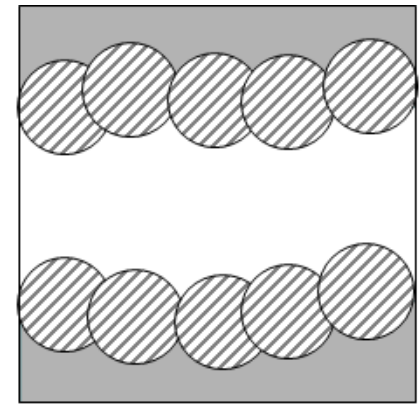

A

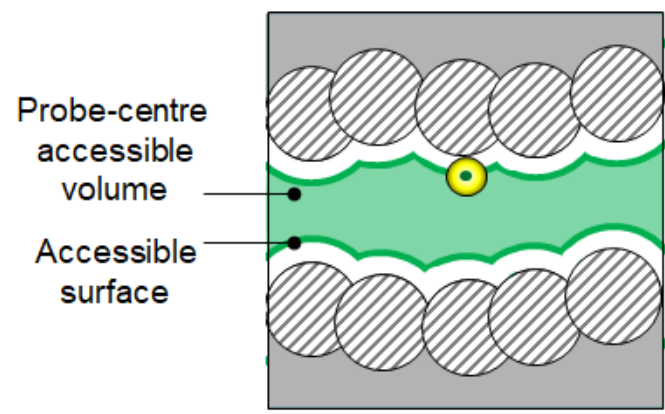

C

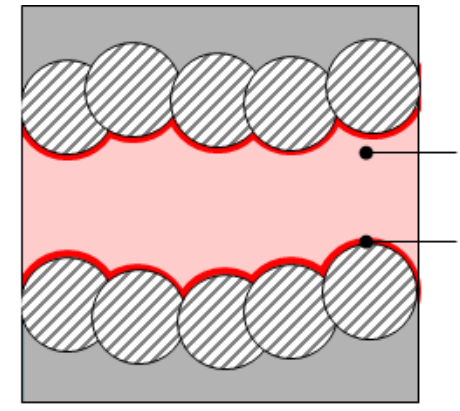

Geometric volume

van der Waals surface

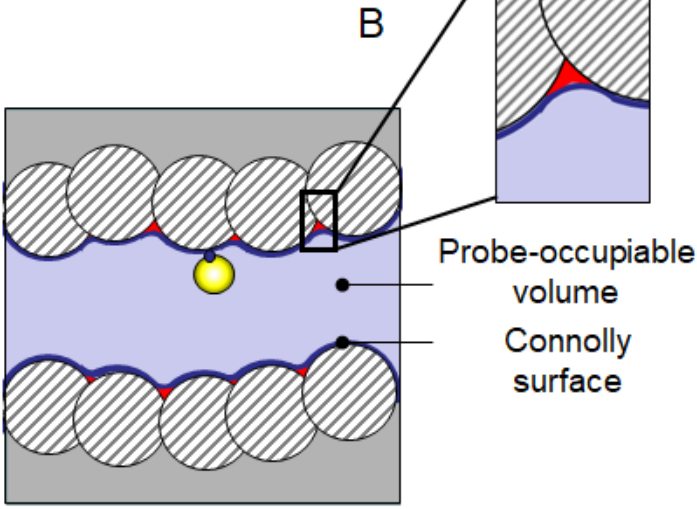

D

Figure 1. Schematic depiction of a porous material and its properties. (A) A model system consists of a material, shown as the area shaded in grey, and a pore spanning the system in the horizontal direction. Atoms of the material at the boundary between the shaded area and the pore are shown as striped circles. (B) The geometric pore volume is defined as the region of the system not occupied by atoms (shown in light red). The boundary between the occupied and empty space is the van der Waals surface, shown in B as the red line. (C) Schematic depiction of the accessible surface: it is the surface formed by the center of the probe particle rolling over the surface of the atoms of the structure, shown as the green line. A region of space enclosed by the accessible surface corresponds to the probe-center accessible probe volume, shown as the green shaded 
area. (D) Schematic depiction of the Connolly surface: it is the surface formed by the tip of the probe particle rolling over atoms of the structure, shown as the dark blue line. A region of space enclosed by the Connolly surface corresponds to the probe-occupiable volume, shown as the blue shaded area. The difference between the geometric volume and the probe-occupiable volume is shown as residual red-shaded areas in panel D (see the inset for more details).

In Figures 1C, D we consider the same process for a probe of finite size. In this case, we generate two surfaces (again, in the case of the 2D schematic, it is two lines): one by the center of the particle, shown as the green line in Figure $1 \mathrm{C}$, and the one by the tip of the particle, shown as the blue line in Figure 1D. The first property has been traditionally called solvent-accessible, or accessible surface in biomolecular studies. The second surface is called the solvent-excluded surface in the biomolecular community, or the Connolly surface. In this article, we are going to use subindex $A C$ for the solventaccessible definition of the surface area $\left(S_{A C}\right)$ and subindex $C$ for the Connolly surface area $\left(S_{C}\right)$.

The physical meaning of the first property, $S_{A C}$, is that it is most closely related to the nitrogen or argon adsorption surface area if we use a probe particle representing properties of these molecules. Indeed, gas-adsorption experimental methods for the determination of the surface area, such as BET, are based on the notion of a layer of molecules forming on the surface (where the capacity of this layer can be extracted from the adsorption isotherms depending on the method) and, given the crosssectional area of the probe molecule, the specific surface area of the material can be determined. Multiple studies have been published on the correlation between the computed areas of a porous material and surface areas extracted from experimental data on real crystals or simulation data on perfect crystals ${ }^{7,26-28}$. Correspondingly, the volume enclosed by this accessible surface is the volume accessible to the probe of a specific size. Following the terminology of Ongari and co-workers ${ }^{29}$, we call this property the probe-center accessible volume, $V_{P C}$. In Figure $1 C$, this is shown schematically as the green-shaded area.

The second property, the Connolly surface, is important for yet another definition of the pore volume. Indeed, in this definition, the pore volume is the volume enclosed by the Connolly surface, shown the blue area in Figure 1D. The physical meaning of this volume is that any point that belongs to any part of the probe atom (not just the center) constitutes the pore volume. It has been argued, that it is this volume that is most consistent with the volume obtained from the experimental nitrogen and argon adsorption and the Gurvich rule, which assumes that the density of the confined liquid in the porous material is equal to the density of the bulk liquid at the same temperature ${ }^{29}$. To distinguish it from the probe-center accessible volume enclosed by the accessible surface, we need to give it a separate name. In the study by Ongari and co-workers and in Zeo++ this volume is called the probeoccupiable (PO) pore volume, $V_{P O}{ }^{29}$. For consistency, we will use the same terminology.

The next property we wish to introduce is the helium pore volume. To understand the nature of this property and its relevance, it is useful to take a brief detour into experimental measurements of adsorption. The property that is determined and, typically, reported in experiments is the excess amount adsorbed $\left(n^{e x}\right)$ :

$$
n^{e x}=n^{a}-V_{\text {dead }} \cdot \rho_{\text {bulk }}(1)
$$

which is the difference between the actual amount of adsorbing species present in the system $n^{a}$ and the amount of gas that would occupy the available space $V_{d e a d}$, as if there was no effect of adsorption. 
Here, $\rho_{\text {bulk }}$ is the bulk gas density at the temperature and pressure of the experiment. This available space, traditionally called dead volume, consists of the volume of the pores $\left(V_{\text {pore }}\right)$ in the material and the volume external to the material sample $\left(V_{\text {external }}\right)$ :

$$
V_{\text {dead }}=V_{\text {pore }}+V_{\text {external }}
$$

To obtain (1), in experiments, the dead volume of the system is determined using a preliminary calibration step based on the helium expansion. For this, helium is introduced in the system at ambient temperature and low pressure. Using the ideal equation of state and the amount of helium in the system, $V_{\text {dead }}$ is determined. The absolute amount adsorbed $\left(n^{a b s}\right)$ is the total number of adsorbing species present in the porous material:

$$
n^{a b s}=n^{e x}+V_{\text {pore }} \cdot \rho_{\text {bulk }}(3)
$$

It has been argued on a number of occasions that the absolute amount adsorbed provides a rigorous basis for the adsorption thermodynamics ${ }^{30,31}$. Moreover, this is also the property used in the process modeling of adsorption and calculated in molecular simulations ${ }^{31}$. Hence, comparison between experimental and simulation studies requires consistent conversion between the excess and absolute properties and the definition of the pore volume, $V_{\text {pore }}$, of the sample. So far, in this section, our definition of pore volume has been based on purely geometric considerations and on some rational way to draw the boundary between what, colloquially speaking, belongs to the solid structure and the remaining empty space. The helium expansion experiment offers another definition of the pore volume:

$$
V_{\text {pore }}=\frac{n_{H e}}{\rho_{H e, b u l k}}(4)
$$

where $n_{H e}$ is the amount of helium present in the pores and $\rho_{H e, b u l k}$ is the density of the bulk helium gas at ambient temperature and pressure. Let us hypothesize that helium is weakly interacting with the porous material and therefore it is not adsorbing. Therefore, inside the pores of the material, it behaves as an ideal gas; applying the ideal gas equation of state to the amount of helium present in $V_{\text {pore }}$ will give us a value that is reasonably consistent with our expectation of what pore volume should be as outlined by some boundary between the solid and the pore space. This is, however, not the case, and particularly in materials with very narrow porosity: several studies have demonstrated that helium does interact with the porous material, although weakly (for a review and discussion of this issue see Brandani et al. ${ }^{31}$ ). As a result, the volume obtained according to Eq. 4 will likely have values not consistent with the alternative definitions of the pore volumes based, for example, on the geometric methods. Ongari et al. ${ }^{29}$, demonstrated that depending on the interactions with the pores of the material, the value of the pore volume estimated in this fashion may be lower or higher than the values of the pore volume defined using the geometric methods.

Hence, as has been argued by Neimark and Ravikovitch ${ }^{32}$, consistent conversion between the experimental and simulation studies requires a consistent definition of the pore volume, regardless of its true physical meaning. In other words, if the calibration with helium expansion was used to obtain $n^{e x}$ in experiments (Eq. 1), then Eq. 3 to obtain absolute amount adsorbed requires $V_{\text {pore }}$ also obtained according to the helium porosimetry and Eq. 4. Similarly, conversion of the simulation values for absolute amount adsorbed to the excess amount adsorbed would require some computational analog of helium porosimetry: 


$$
n_{\text {sim }}^{e x}=n_{\text {sim }}^{a b s}+V_{H e, s i m} \cdot \rho_{\text {bulk }}(5
$$

where $n_{\text {sim }}^{a b s}$ is the absolute amount adsorbed in the simulations and $V_{H e, s i m}$ is the simulated helium pore volume. Indeed, this approach to obtain and report simulated excess adsorption isotherms has been adopted in many previous publications ${ }^{33,34}$.

The essence of the computational helium porosimetry is to obtain $V_{\mathrm{He}, \mathrm{sim}}$ in simulations. In principle, we could use the standard grand canonical Monte Carlo simulation to obtain the amount of helium adsorbed at the specified temperature and pressure of the bulk phase. However, the fact that at ambient temperature and low pressure we are located in the Henry's regime of adsorption for helium simplifies the analysis. In the Henry's law regime, the amount adsorbed is:

$$
n_{H e}=\rho_{H e, b u l k} \int_{V} e^{-\frac{U(r)}{k T}} d \boldsymbol{r}(6)
$$

where $U(\boldsymbol{r})$ is the interaction potential experienced by a helium atom at location $\boldsymbol{r}$ within the system and the integration takes place over the volume of the simulation cell. From Eqs. 4 and 6, the $V_{\mathrm{He}, \mathrm{sim}}$ becomes:

$$
V_{\text {pore }}=\int_{V} e^{-\frac{U(r)}{k T}} d \boldsymbol{r}=V \cdot\left\langle e^{\left.-\frac{U(r)}{k T}\right\rangle(7)}\right.
$$

where $V$ is the volume of the system. The property in the angle brackets is the average Boltzmann factor, which is estimated by placing a probe helium atom in random locations throughout the system and estimating its interactions with the material. From this analysis, it is clear that the helium pore volume estimated according to Eq. 7 will depend on temperature (although weakly when close to the ambient range), and on the interaction parameters of the helium atom used and the atoms of the material. It is also important to note that the Eqs. 6 and 7 can be also easily linked to the Henry's constant of adsorption. Using ideal gas equation of state for helium, one obtains:

$$
\frac{n_{H e}}{V}=\frac{P}{R T}\left\langle e^{-\frac{U(r)}{k T}}\right\rangle(8)
$$

leading to the Henry's constant:

$$
K_{H}=\frac{\left\langle e^{\left.-\frac{U(r)}{k T}\right\rangle}\right.}{R T} \text { (9) }
$$

From the expressions above it is clear, that the calculation invoved in obtaining the helium pore volume can be equally used to obtain the Henry's constant of adsorption for other gases of interest, using appropriate interaction parameters.

The final property we wish to introduce is the pore size distribution (PSD). Crystalline and disordered porous materials (such activated carbons) can be seen as a system of pores of different sizes. The total volume of all pores is equal to the cumulative pore volume of the system. PSD and how pores are topologically arranged governs adsorption and transport properties of a porous material, and it is vital in the characterization of their structure. In experiments, a PSD is obtained by interpreting the nitrogen or argon adsorption isotherms measured at cryogenic conditions as a cumulative result of adsorption in a system of independent cylindrical, slit, or spherical pores of different sizes. In the modern approaches to characterization, the classical Density Functional Theory (DFT) is employed to generate a kernel of isotherms for individual pores of specific width or diameter. This kernel of the isotherms, combined with the experimental adsorption isotherm, is then used to 
obtain the frequency with which pore of each size is present in the system, or, in other words, the pore size distribution. Although it is now a standard approach in the characterization of porous materials using physical adsorption experiments, it comes with several challenges. Firstly, the link between adsorption in the whole sample and adsorption in individual pores is established through the so-called Adsorption Integral Equation (AIE). The AIE corresponds to a Fredholm integral equation of the first kind, commonly known to be both an ill-posed and ill-conditioned problem. This leads to either no solution or to an infinite number of possible solutions which in turn are extremely sensitive to small changes in the input. Therefore, to obtain reliable PSD by solving the AIE, advanced techniques, based for example on regularization, need to be employed. Secondly, the current kernels have been derived predominantly for the systems reflecting chemistry and properties of activated carbons and certain zeolites. Direct application of these kernels to other classes of materials such as MOFs should be approached with caution and the development of more specialized kernels for MOFs is an ongoing area of research ${ }^{35}$. Finally, only for a few materials, a picture of independent pores of simple geometry is realistic, and most of the materials would feature a network of pores. Interpretation of adsorption isotherms and in particular adsorption hysteresis in terms of network connectivity of pores is still an ongoing area of research, although significant progress has been achieved in recent twenty years. For a more comprehensive review of the adsorption characterization methods and application of DFT, we refer the reader to the excellent articles by Neimark and coworkers ${ }^{36}$ and by Thommes and co-workers ${ }^{37}$.

For modeling porous materials we have two options to obtain PSD. We can simulate a nitrogen or argon adsorption isotherm and interpret the results using the existing methods based on the AIE inversion (using either DFT kernels or bespoke kernels from additional molecular simulations). Alternatively, we can use geometric methods, which will attempt to allocate each point of the porous space to a pore of a particular size. One particular method implemented in PB v4.0 has been originally employed by Gelb and Gubbins in the characterization of model porous glasses ${ }^{8-10}$. We will describe the method in more detail in section 2.3 on the algorithms. Here, it suffices to say that for the case of mesoporous materials, Gelb and Gubbins observed surprisingly reasonable correlation between the geometric PSDs and the PSDs from the physical adsorption characterization, although, clearly, the methods are based on completely different principles.

In the case of MOF and other crystalline materials, the geometric methods are expected to identify pores of a specific well-defined size and the PSD should look like a collection of discrete peaks. This information is useful to understand the dimensions of the existing channels and cages and as a part of the digital identity of a MOF - we can envision that selection and screening of MOFs within a database can be done to identify MOFs with a profile of cages and channels of a certain size. Attempts to connect geometric PSDs to specific adsorption behavior (for example, presence of cages of a certain size should lead to the corresponding number of steps in the adsorption isotherm) proved to be, however, difficult for microporous materials and it is still research in progress ${ }^{38}$. In section 2.3, we will illustrate how geometric methods would tend to interpret cage-like porous space of MOFs, and in the results section, we will provide several examples of this function for materials in the case studies.

\subsection{Network accessible and network non-accessible properties}

Consider a model network of pores shown in Figure 2. It consists of a single pore spanning the system in the horizontal direction and also two spherical cavities. One cavity is completely isolated from the outside space (i.e. closed porosity) and another one is connected to the main pore via a narrow 
channel. This simple pore network allows us to introduce the notion of network accessibility and several other related concepts. Indeed, depending on the size of the probe, various subregions of the pore space shown in Figure 2 will be available for the probe to explore. A point-size probe entering the system on the left through the main pore will be able to traverse the system from left to right and also explore the side channel and the spherical pore connected to it. The pore in the top left corner of the system cannot be reached via a continuous, physically meaningful walk by a probe of any size. A probe of small size may be able to go to the side cavity in the top right corner via the channel if the channel is wide enough. The region of space accessible to the center of the probe via physically connected space is shown in yellow in Figure 2A. For a probe of a larger size, as shown in Figure 2B, the side channel may be too narrow to pass and the side pore is not accessible for it to explore. The regions that the center of the blue particle can reach via a physically meaningful walk are shown as the light blue shaded areas in Figure 2B. The largest probe that can cross the simulation cell in at least one dimension via a diffusive pathway is said to correspond to the Pore Limiting Diameter (PLD), as illustrated in Figure 2D. Hence, we can define regions of porous space as network-accessible to a probe of a particular size if they form a percolated network spanning the system in at least one dimension. In other words, network-accessible space is formed by all pores inside the material, which a probe molecule can reach and diffuse through if it was an actual experiment.

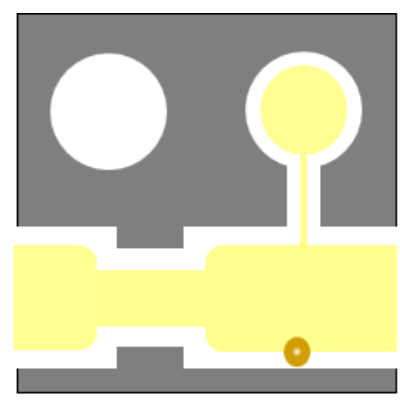

A

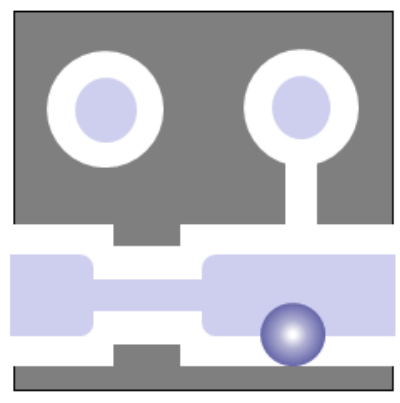

C

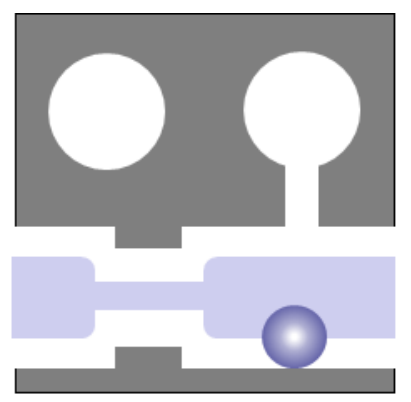

B

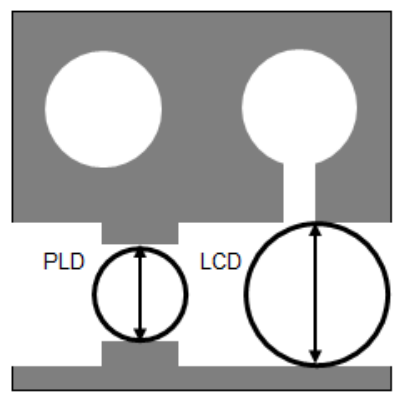

$\mathrm{D}$

Figure 2. Schematic illustration of the network-accessible and non-accessible properties. (A) For a small probe particle, shown as the yellow sphere, the area shaded light yellow is network-accessible to the center of the probe. The spherical cavity in the top left corner of the system is not network-accessible to the probe. (B) For a probe of a different (in this case, larger) size, the network-accessible regions will be different, here shown as the light blue shaded area. (C) This panel illustrates the total probe-center accessible volume for the blue particle. (D) This schematic illustrates the concepts of the Pore Limiting Diameter (PLD) and Largest Cavity Diameter (LCD). 
Each of the properties defined in 2.1 can be calculated considering either all the pores in the system, even the ones that are isolated or inaccessible to a probe of a particular size, or only using the percolated network - in other words, network-accessible regions. For example, consider the accessible surface area, $S_{A C}$. For this property, we use a molecular probe corresponding to an atom of nitrogen. As has been discussed before, this is the surface enclosing the probe-center accessible volume. If we take network accessibility of the porous space into account, it is the blue shaded area in Figure 2B as discussed before. However, if the network accessibility is not taken into account, it leads to a different picture: the volume accessible to the center of the probe, in this case, is shown in Figure 2C. It includes regions within the two spherical cavities, which are large enough to accommodate the blue particle, but are not reachable by a diffusive pathway. The surface area will correspond to the boundaries between the blue regions and the white regions in the two schematics and it will have different values depending on the approach (network-accessible vs. not networkaccessible).

In experiments, obviously, all properties measured via gas adsorption correspond to the networkaccessible regions of the porous space. Therefore, for consistent comparison of the properties, we need to distinguish between network-accessible and not network-accessible properties. Often, these have been referred to as open and closed porosity, respectively. We note that in defining these properties, it is important to state with respect to what probe the accessibility is considered. Not all combinations of properties actually are physically relevant or can be compared to the experimental counterparts. For example, for the surface area, it makes sense to define $S_{A C, A\left(N_{2}\right)}$ as accessible surface area obtained specifically for a nitrogen-accessible network (Figure 2B), as this is what would be measured in the actual gas adsorption experiments, and $S_{A C, T\left(N_{2}\right)}$ as the total accessible surface area (Figure $2 \mathrm{C}$ ).

Table 1 summarizes the properties reported by PB v4.0. Each property can be either of the total (second subindex $T$ ) or network-accessible type (second subindex, $A$ ). In these definitions, we use a specific combination of the property and the probe, and therefore additional information on the nature of the probe is not needed: for example, $S_{A C, A\left(N_{2}\right)}$ is simply $S_{A C, A}$. To avoid cumbersome full name for "network-accessible accessible surface area", we make a convention here that, unless specified otherwise, the surface area term describes the accessible surface area. This convention allows us to call $S_{A C, A}$ property the network-accessible surface area and $S_{A C, T}$ the total surface area, respectively.

Table 1. Properties reported by PB v4.0.

\begin{tabular}{cccc}
\hline Property & Probe & Network & Notation \\
\hline Accessible surface area & Nitrogen & Nitrogen & $S_{A C, A} ; S_{A C, T}$ \\
Geometric volume & Point & Point & $V_{G, A} ; V_{G, T}$ \\
Probe-occupiable volume & Nitrogen & Nitrogen & $V_{P O, A} ; V_{P O, T}$ \\
Helium pore volume & Helium & Helium & $V_{H e, A} ; V_{H e, T}$ \\
\hline
\end{tabular}

In addition to the properties in Table 1, PB v4.0 also reports the PLD, the number of dimensions in which the system is percolated and the PSD. If the PLD is smaller than the size of the nitrogen probe, naturally the code will deem this material is not accessible to nitrogen and report zero values for $S_{A C, A}$ and $V_{P O, A}$. Similarly to the properties in Table 1, the PSD is reported for the network-accessible 
subvolume of the system and for the total volume accessible to the center of the nitrogen probe. Finally, the largest pore in the structure can be characterized by the largest cavity diameter (LCD), also illustrated in Figure 2D. In Zeo++, this property is called the largest included sphere (LIS). Also, in Zeo++, an additional property is identified, which is the largest included sphere along the percolated pathway. The LCD should be consistent with the largest pore size reported in PSD.

\subsection{Methods and algorithms}

In this section, we turn our attention to the methods and algorithms involved in the calculation of the properties defined above. In the first step of the PB v4.0 code, the system is divided into small cubelets and, in the preliminary calculation, the distance between the centers of all cubelets and centers of the atoms of the structure is calculated and stored for later use. Using this lattice of cubelets, we can explore pore volume accessible to a center of a particular probe. For this, we first identify all the cubelets, such that if the probe particle is placed in the center of the cube, it does not overlap with any atoms of the structure. Mathematically, this condition can be expressed as:

$$
\left|\vec{r}_{i}-\vec{a}_{j}\right|>\sigma_{i j} / 2 \text { for } \forall j \in \mathbf{J}(10)
$$

where $\vec{r}_{i}$ is the coordinate of the center of cube $i, \vec{a}_{j}$ is the location of atom $j, \mathrm{~J}$ is a set of all atoms in the system, and $\sigma_{i j}$ is the collision diameter between the probe $i$ and atom $j$ of the structure. Within the same lattice subroutine, the code also calculates and stores the distance between the center of the cube and the surface of the atoms: $r_{i j, S}=\left|\vec{r}_{i}-\vec{a}_{j}\right|+\sigma_{j} / 2$. This property will be employed later in the section.

Using a lattice representation of the porous space, PB v4.0 invokes the Hoshen-Kopelman algorithm ${ }^{39}$ to explore the percolation of the porous space with respect to the probes of different types (e.g. point probe, nitrogen atom, helium atom). This is schematically depicted in Figure 3 . In panel $B$, the system shown in panel $A$ is divided into a lattice of small cubelets. Cubelets shaded in grey are accessible to the blue probe particle as this leads to no overlaps with the atoms of the structure, shown as the striped grey particles. The cluster of lattice sites in the middle of the system in panel B forms a percolated pathway for the blue probe particle in the vertical direction - this cluster corresponds to the network-accessible probe-center volume $V_{P C, A}$, whereas the whole set of cubelets shaded grey forms the total probe-center volume $V_{P C, T}$. Pore limiting diameter is identified as the largest probe for which a percolating lattice cluster exists, spanning the system in at least one dimension. Along with the PLD, PB v4.0 will also return in how many dimensions percolation with the current PLD has been detected $(1,2$ or 3$)$. If in Figure 3B the blue particle is the largest probe that can traverse across the system, it is the size of this probe that corresponds to the PLD. In the case of the model material shown in Figure 2D, it is the constriction of the main pore that defines the PLD. The fortran maxval function applied to the array storing the distances between the centers of the cubelets and the surfaces of the atoms identifies the site corresponding to the largest value of the distance stored. This value corresponds to the Largest Cavity Diameter (LCD), also shown in Figure 2D, schematically.

To calculate the accessible surface area, typically, a Monte-Carlo algorithm is invoked. This is schematically depicted in Figure $3 \mathrm{C}$, with the probe particle shown as a dashed green circle and the atoms of the structure shown as grey striped circles as before. Points are generated randomly on a 
surface of a sphere of radius $r=k\left(\frac{\sigma_{i}+\sigma_{p}}{2}\right)$, shown in Figure $3 \mathrm{C}$ as the green circle, where $\sigma_{i}$ is the diameter of atom $i, \sigma_{p}$ is the diameter of the probe particle and $k$ is a coefficient which by default is equal $2^{1 / 6}$ in PB $v 4.0$. This value of the coefficient makes the distance $r$ correspond to the location of the Lennard-Jones potential minimum. Although other conventions are possible (e.g., $k=1$, corresponding to the location where the Lennard-Jones potential between the two particles is equal to zero), however, we believe the approach adopted here more accurately reflects the physical location of a monolayer adsorbed on the surface of the material. For each point, the test is then performed to check whether it is within the collision distance $r$ with any other atoms of the structure, and if it is not, it counts as a point on the accessible surface, shown in Figure $3 \mathrm{C}$ as green dots. Points that fail this test are shown in red. The accessible surface area associated with the adsorbent atom $i$ under consideration is then given by: $a_{i}=f \cdot\left(4 \pi r^{2}\right)$ where $f$ is the fraction of green points in the trial. Schematically, the proportion of the surface area that is not accessible to the probe particle is shown as the red arc in Figure 3C. This is indeed the algorithm implemented within PB v4.0. The lattice site representation of the space offers two additional functionalities: firstly, the check on whether a point on the test sphere belongs to an accessible surface does not require an additional distance calculation, just a look-up in the table for the cubelet to which the generated point belongs. Furthermore, as we discussed before, the accessible surface is the boundary between the probecenter accessible volume and the rest of the space. In principle, the area of this boundary can be simply estimated from the surface area of the cubelets belonging to the probe-center volume. For this, one simply needs to count all the faces of the cubelets within $V_{P C}$ that are not shared with other cubelets within $V_{P C}$ (i.e., they are exposed, as the cubelet sits on the boundary). This should provide a significant speed-up of the code, however, this has not been implemented yet.
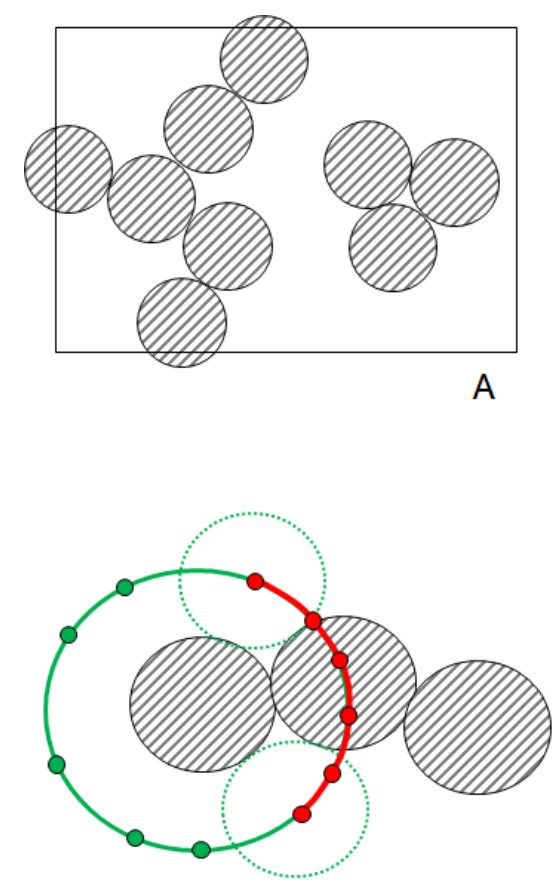

C
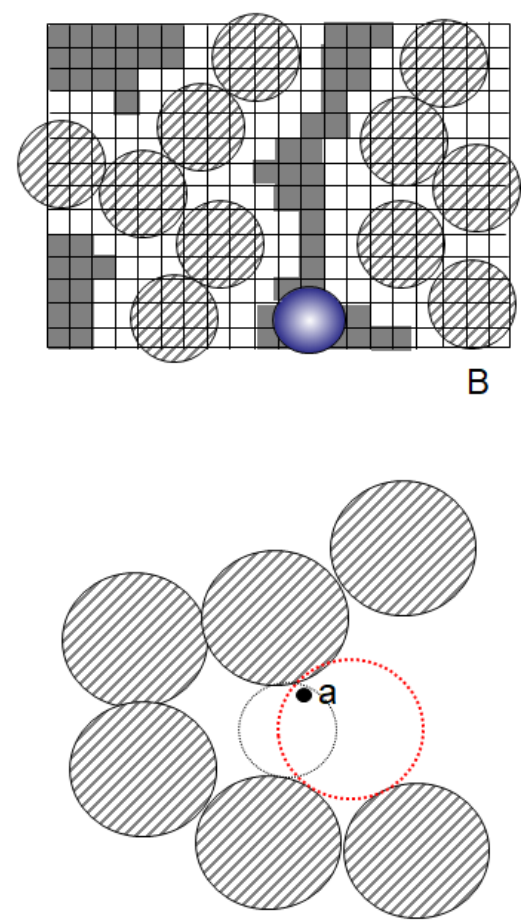

$\mathrm{D}$

Figure 3. Schematic illustration of some of the concepts involved in the PB v4.0 algorithms. (A, B) A model system consisting of atoms, represented as striped circles (A), is divided into cubelets (B), with the grey 
cubelets representing accessible cubelets to the center of the probe. Percolation algorithms are then used to identify a set of cubelets forming a continuous pathway across the system, as shown in (B) for the blue particle. (C) Illustration of the Monte Carlo algorithm to calculate the surface area of the structure. For each atom, the algorithm obtains the proportion of the points sitting on the accessible surface of the atom (green circle), without overlapping with other atoms of the structure. (D) Geometric PSD calculation. A point "a" belongs to the pore shown as the red dashed circle: this is the largest pore containing the point without an overlap with the atoms of the structure.

Calculation of the Connolly-surface enclosed volume $V_{P O}$ is closely related to the calculation of the pore size distribution, and therefore it would be logical to introduce this property and the algorithm first. The algorithm effectively consists of two nested loops of random trials. First, a random point in space not overlapping with the atoms of the structure is generated (point "a", in Figure 3D). In the second cycle, another set of random points space is generated. Each of these points is the center of a sphere, which is assigned the maximum diameter possible without overlapping with the structure of the atoms. Figure 3D shows two dashed circles as examples of this trial. The largest diameter identified in the second trial that contains the first random point corresponds to the largest pore to which the point belongs (the red dashed circle in Figure 3D). All pores of this and smaller diameter contain this point, which upon completion of the cycle produces the cumulative pore size distribution. The derivative of this function with respect to the radius or diameter of the pore produces the conventional pore size distribution.

The lattice representation of the space offers few simplifications of this algorithm. A cubelet is selected at random from the geometrical subset, $V_{G}$, this is equivalent to randomly choosing a point in space in the off-lattice version. The distance between this cubelet and all other cubelets has been already pre-calculated (or can be easily looked up simply using the lattice indices). As has been already mentioned, in the preliminary calculations, we also computed the distances between the lattice sites and the positions of the atoms constituting the structure of the material. Therefore, using a simple sorting algorithm, it is easy to find the cubelet, the center of which is also the center of the largest sphere that contains the center of the original cubelet from the $V_{G}$ lattice. The total volume of the pores identified in this fashion corresponds to the volume included by the Connolly surface formed by the tip of the nitrogen probe, as we defined earlier, the probe-occupiable volume, $V_{P O}$. If we restrict the sorting algorithm to only cubelets belonging to the network-accessible probe-center accessible volume, $V_{P C, A}$, then the pore size distribution will also correspond to the network-accessible region of the porous space and the total volume of these pores will correspond to the network-accessible, probe-occupiable volume, $V_{P O, A}$.

What are then the PSDs one might expect for the MOF materials? To understand this, it is useful to explore the structure of the PSDs that the algorithms described above would generate for simple pore geometries such as a spherical cage, a slit, or a square pore. This is schematically depicted in Figure 4. Indeed, the PSDs for the spherical cage and the slit pre would produce an expected $\delta$-function located at the diameter of the pore. A square pore represents a more interesting case. An intuitive guess would suggest also a PSD with a single peak corresponding to the largest pore that can be inscribed in a square. This however neglects the existence of the regions in the corners of the pore, which according to the algorithms described above would be assigned to pores of a smaller size. The corner of a square represents a wedge geometry and this should give rise to a continuous tail of diminishing pore sizes in the PSD. Many of the MOF materials feature cube-like and square like pores 
(e.g., IRMOF series of materials) and therefore similar trends for PSDs would be observed for these MOFs as well.
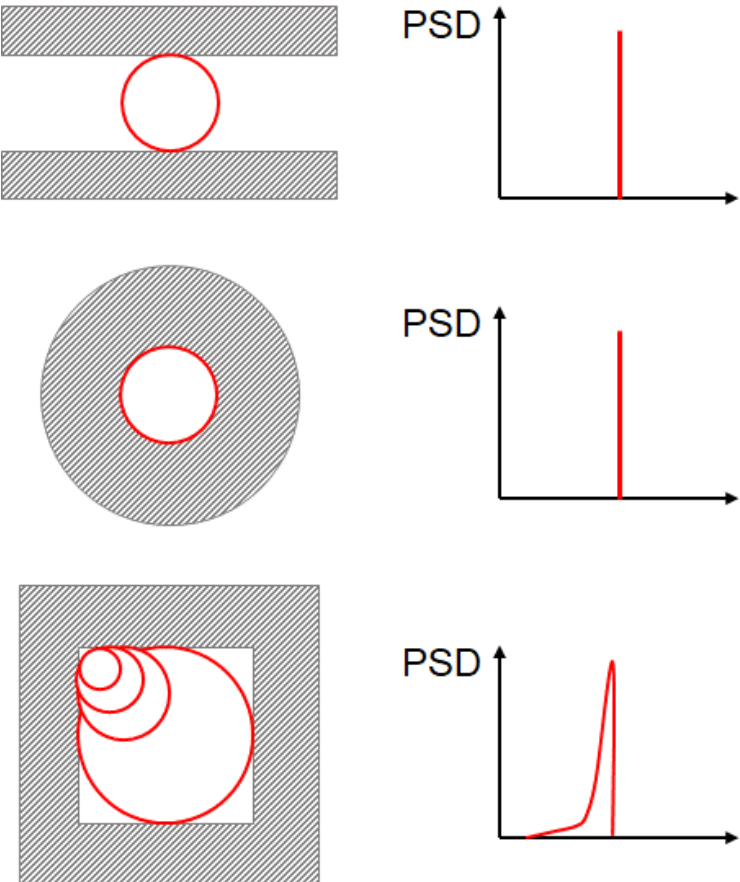

Figure 4. Schematic illustration of the geometric Pore Size Distribution in model structures.

\section{Analysis of the textural properties of MOFs}

\subsection{Case studies: HKUST-1, IRMOF-1, ZIF-8}

Let us now consider the application of PB v4.0, Zeo++ and RASPA to three quintessential and wellknown MOF materials: HKUST-1, IRMOF-1 and ZIF-8. In the S1 section of the Electronic Supplementary Information (ESI) file prvide complete description of the files and parameters required to setup PB v4.0 simulations. Figure 5 shows molecular structures of the materials under consideration. Table 2 summarizes the results for PB v4.0 and Zeo++. Overall, they exhibit a high level of agreement and consistency with each other. This is very reassuring for the community working on the material informatics and computational screening studies and do rely on these two codes.
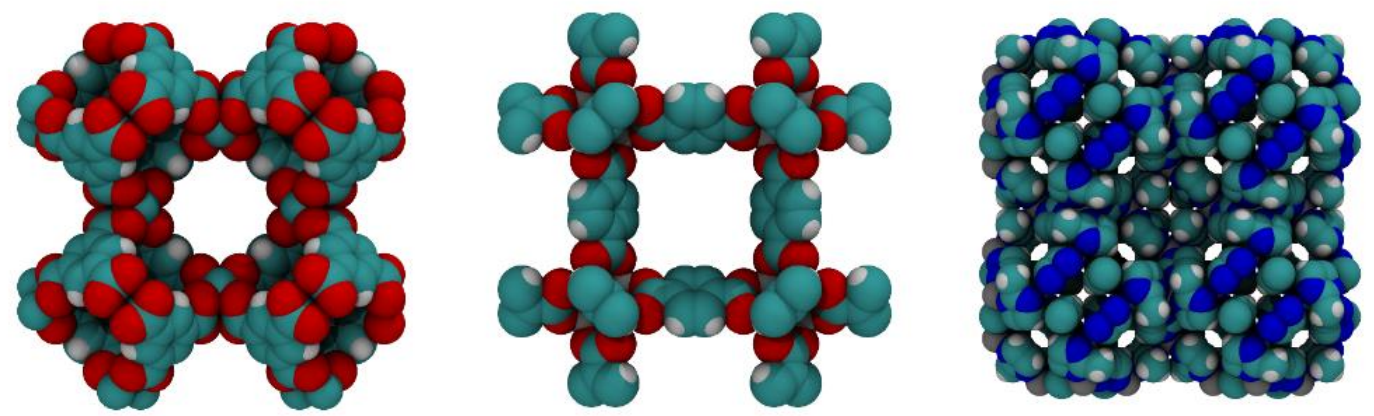
Figure 5. Molecular visualizations of HKUST-1 (left), IRMOF-1 (center), and ZIF-8 (right). This image was made with VMD software ${ }^{40}$.

Table 2. Comparison of the results from PB v4.0 and Zeo++ for HKUST-1, IRMOF1, and ZIF-8. Showing density, pore-limiting diameter (PLD), largest cavity diameter (LCD), network-accessible surface area $\left(S_{A C, A}\right)$, total surface area, $\left(S_{A C, T}\right)$, network-accessible probe-occupiable volume, $\left(V_{P O, A}\right)$, total probe-occupiable volume, $\left(V_{P O, T}\right)$ and $C P U$ time.

\begin{tabular}{ccccccc}
\hline & \multicolumn{2}{c}{ HKUST-1 } & \multicolumn{2}{c}{ IRMOF-1 } & \multicolumn{2}{c}{ ZIF-8 } \\
\hline Property & PB & Zeo++ & PB & Zeo++ & PB & Zeo++ \\
\hline Density $\left[\mathrm{g} / \mathrm{cm}^{3}\right]$ & 0.884 & 0.884 & 0.593 & 0.593 & 0.923 & 0.924 \\
PLD $[\AA]$ & 6.380 & 6.337 & 7.800 & 7.773 & 2.860 & 3.073 \\
LCD $[\AA]$ & 12.86 & 12.99 & 15.03 & 15.03 & 11.42 & 11.40 \\
$S_{A C, A}\left[\mathrm{~m}^{2} / \mathrm{g}\right]$ & 1808.09 & 1801.02 & 3400.07 & 3448.82 & 0.00 & 0.00 \\
$S_{A C, T}\left[\mathrm{~m}^{2} / \mathrm{g}\right]$ & 1860.87 & 1853.46 & 3459.99 & 3448.82 & 1168.58 & 1156.51 \\
$V_{P O, A}\left[\mathrm{~cm}^{3} / \mathrm{g}\right]$ & 0.759 & 0.72 & 1.30 & 1.27 & 0.00 & 0.00 \\
$V_{P O, T}\left[\mathrm{~cm}^{3} / \mathrm{g}\right]$ & 0.762 & 0.722 & 1.304 & 1.270 & 0.517 & 0.501 \\
CPU time $[\mathrm{s}]$ & 116.847 & 683.857 & 92.104 & 672.437 & 347.058 & 2714.391 \\
\hline
\end{tabular}

In addition, Table 3 compares the results from PB v4.0 and RASPA. Using RASPA, it is possible to calculate the accessible surface area and helium pore volume fraction, $F_{H e, T}=V_{H e, T} / V$. Network connectivity analysis is not performed in RASPA and, therefore, all the properties reported are total properties, and not network-accessible properties. On a further note, in the case of Zeo++, the helium pore volume is not calculated according to Eq. 7. Instead, it is based on the hard-sphere helium atom probe, making this volume helium probe-center volume in our definition (either total or networkaccessible). Hence, we do not provide a comparison of this property between PB V4.0, RASPA, and Zeo++, as it should not be expected to produce an agreement. We will, however, return to this comparison in the next section for the complete set of MOF materials in this study. In the case of CPU time, the results provided for RASPA correspond to the summative time required to obtain properties listed in Table 3. It is important to emphasize that the CPU times listed in Tables 2 and 3 are setupspecific, and they depend on the actual size of the unit cell used and the number of cycles involved for sampling the properties. On https://github.com/SarkisovGroup/PoreBlazer, we provide complete setups used to obtain the results in Tables 2 and 3, so the reader knows precisely under what conditions this performance is observed. Section S2 in the ESI provides a more additional analysis of the performance of the code and the sensitivity of the PB v4.0 results to the size of the grid.

Table 3. Comparison of the results from PB v4.0 and RASPA for HKUST-1, IRMOF1, and ZIF-8. Showing density, Helium pore volume fraction $\left(F_{H e, T}\right)$, Total surface area $\left(S_{A C, T}\right)$ and CPU time.

\begin{tabular}{ccccccc}
\hline & \multicolumn{2}{c}{ HKUST-1 } & \multicolumn{2}{c}{ IRMOF-1 } & \multicolumn{2}{c}{ ZIF-8 } \\
\hline Property & PB & RASPA & PB & RASPA & PB & RASPA \\
\hline Density $\left[\mathrm{g} / \mathrm{cm}^{3}\right]$ & 0.884 & 0.884 & 0.593 & 0.593 & 0.923 & 0.924 \\
$F_{H e, T}[-]$ & 0.739 & 0.751 & 0.820 & 0.831 & 0.527 & 0.538 \\
$S_{A C, T}\left[\mathrm{~m}^{2} / \mathrm{g}\right]$ & 1860.87 & 1857.54 & 3459.99 & 3435.09 & 1168.58 & 1166.09 \\
CPU time $[\mathrm{s}]$ & 116.847 & 420.33 & 92.104 & 202.12 & 347.058 & 5183.21 \\
\hline
\end{tabular}


Figure 6 shows the PSDs for three structures. Although there are some variations in the details of the curves, the three codes return the same number of peaks and the same location of peaks within $0.2 \AA$, the lattice precision of PB v4.0. These PSDs show the expected profiles of distinct peaks, with a tail at lower pore sizes.
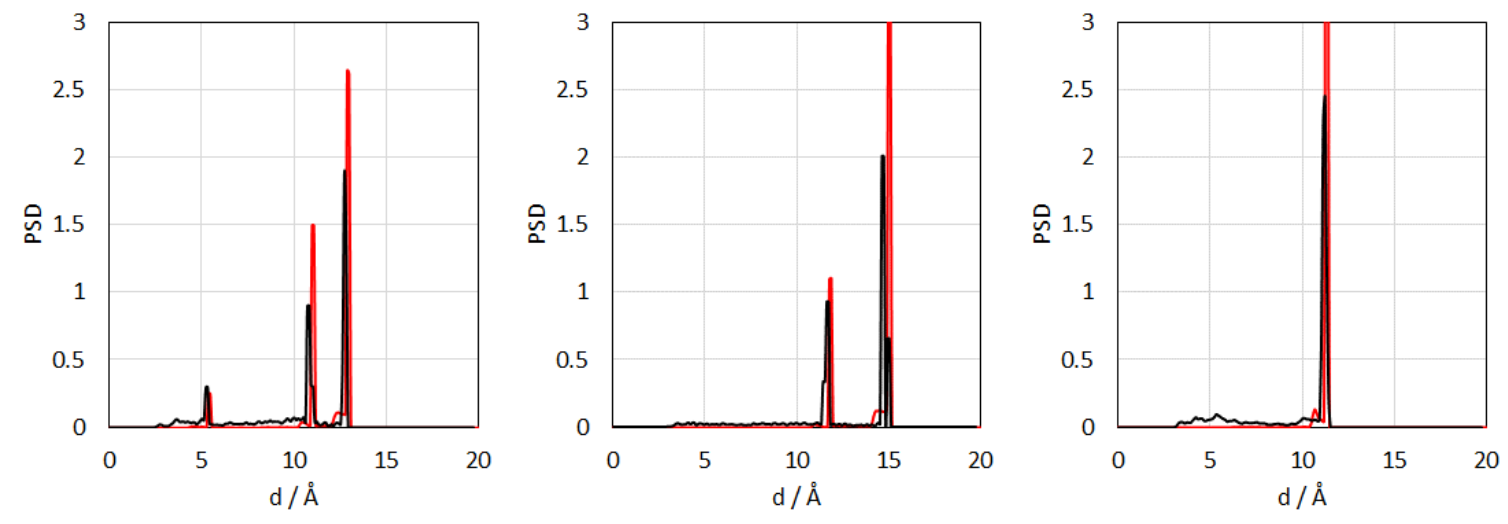

Figure 6. PSDs for HKUST-1 (left), IRMOF-1 (center),and ZIF-8 (right). Red lines are the Zeo++ results; black lines are the PB v4.0 results. For HKUST-1 and IRMOF-1, PSD is the network-accessible property; for ZIF-8 the network-accessible probe-occupiable volume is zero, and therefore PSD is calculated on the whole porous space (total property).

\subsection{Geometric analysis of the CDS MOF database}

We now turn our attention to the calculation of MOFs stored within the CSD MOF subset. The nondisordered CSD MOF subset v5.40 (May 2019) contains ca. 70,000 MOFs'22. From this subset, we filtered out MOFs with structural disorder, partial occupancy issues or missing framework hydrogens, using the bash script and methodology provided by Fairen-Jimenez and co-workers. ${ }^{25,}{ }^{41}$ For the complete tutorial about how to use the CSD MOF subset, we refer the reader to the work from Li et $a .^{38}$ The filtering step produced ca. 57,000 structures. In the next step, we removed the non-bonded solvent molecules as well as those bonded to open metal sites using the CSD Python API, according to the procedure by Li et al. ${ }^{41}$ Calculations on these ca. 57,000 structures revealed that only ca. 12,000 MOFs have non-zero surface area (in other words, they are porous). Therefore, the structure analysis presented here was performed on this subset of structures - 12,052 MOFs for PB v4.0 vs. Zeo++ comparison, and 12,081 MOFs for PB v4.0 vs. RASPA comparison. The difference in numbers is due to the fact that 29 MOFs from the larger set of 12,081 structures had technical issues/errors in Zeo++.

When comparing the results from PB v4.0, Zeo++ and RASPA, we did expect the existence of a subgroup of MOFs for which these codes do not produce a consistent picture. Our philosophy in this section is to first present the results for all the properties of interest (section 3.3.1) in the same way they emerged from our calculations, giving some preliminary comments on the observed trends, distribution of properties and differences in the predictions; then, to provide a more in-depth analysis on what properties exhibit the most significant deviations, possible scenarios that may lead to the disagreements in the predictions, further investigation of the characteristics of the MOFs in the set for which the agreement is acceptable and in the set of MOFs for which results are not consistent. 


\subsubsection{Results and comparison for ca. 12,000 MOFs}

Figure 7 shows the parity plots for the different textural properties - pore limiting diameter, PLD; largest cavity diameter, LCD; total and network-accessible surface area, $S_{A C, T}$ and $S_{A C, A}$; and the and network-accessible total probe-occupiable volume, $V_{P O, T}$ and $V_{P O, A}$ calculated from PB v4.0 and Zeo++ and they distribution from PB v4.0. Figure $\mathrm{S} 3$ in the ESI shows a similar comparison between PB v4.0 and RASPA. With the color bar, we indicate the population of the properties in the different range of values. First, for PLD and LCD, overall, the results show a high level of consistency across the database of materials. From the color bars and from the distribution of the properties, it is evident that only a few materials exhibit PLDs and LCDs above $20 \AA$ (not shown), while the majority of the materials exhibit both PLDs and LCDs below $10 \AA$ (Figs. 7a-b).

When looking at the total surface area, there is again a high level of consistency between the three codes (Fig. 7c and Fig. S3 in the ESI). A different picture, however, emerges for the network-accessible surface area (Fig. 7d). This property depends on how the accessibility of the pore network is calculated and, given the differences in the algorithms between Zeo++ and PB v4.0, it is not surprising that this property is more sensitive to the code used. Interestingly, there is a set of MOFs $(\sim 2,000)$ showing zero network-accessible surface area in Zeo++ and non-zero values in PB v4.0; it manifests itself as a horizontal line of points in the figure. We will return to a more comprehensive analysis for the reasons for these deviations in section 3.2.2 once we present the results on the remaining properties (pore volumes and their distributions). The differences in the distribution of the total and networkaccessible surface areas obtained from PB v4.0 is remarkable. The most striking feature is the large number of MOFs that have a surface area close to zero (they are not strictly zero as materials with strictly zero surface area have been already eliminated in the preliminary screening of the 57,000 MOFs). This implies that the CSD MOF subset, i.e. the database compiling all the reported MOFs from the literature, is dominated by materials with low surface areas and porosities. Even within this smaller subset of 12,052 porous MOFs, more than 3,000 have total surface areas below $50 \mathrm{~m}^{2} / \mathrm{g}$, and only 2,455 MOFs have total surface areas above $1000 \mathrm{~m}^{2} / \mathrm{g}$ - i.e. only about $20 \%$ of $c a .12,000$ MOFs. However, we also need to be aware that some materials will appear in this analysis as non-porous, whereas in reality, they are porous, with windows close to the size of the nitrogen molecules. A certain degree of structural flexibility, not considered in the methods based on the assumption of the rigid framework, allows nitrogen to adsorb in the actual experiments. ZIF-8 is one of the most prominent examples of the materials belonging to this category ${ }^{42}$. 

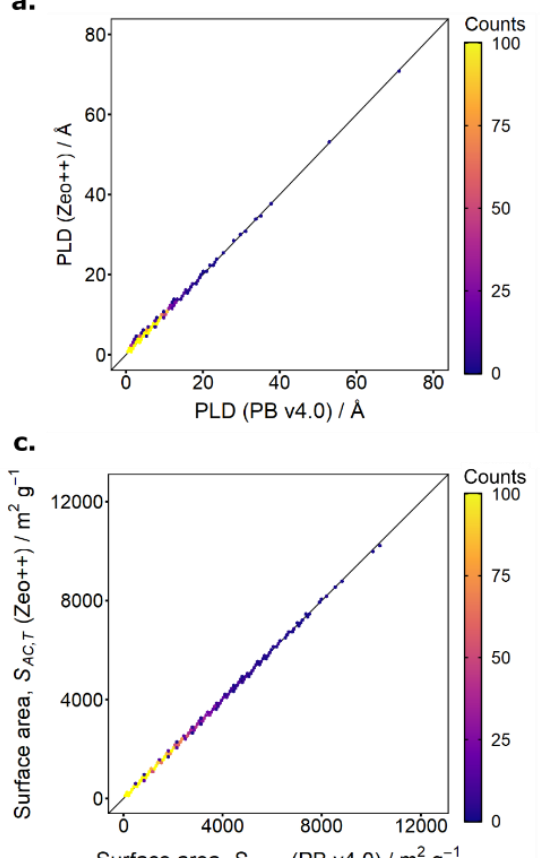

Surface area, $S_{A C . T}\left(\mathrm{~PB}\right.$ v4.0) $/ \mathrm{m}^{2} \mathrm{~g}^{-1}$

e.

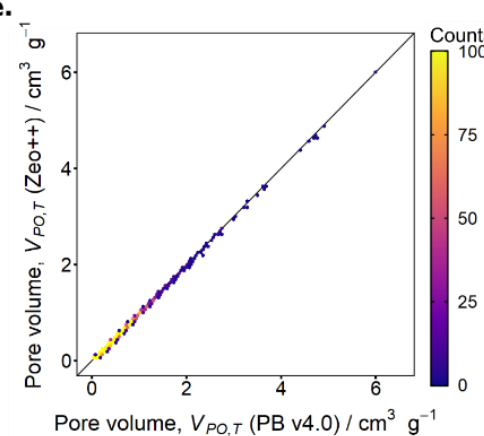

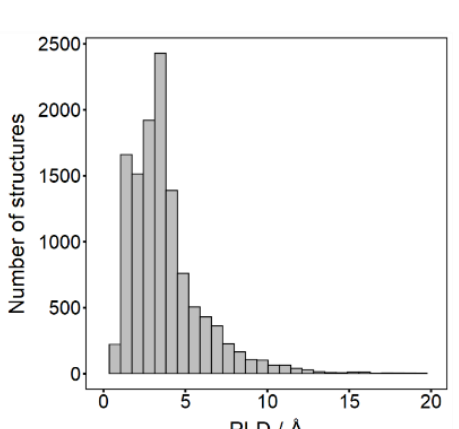

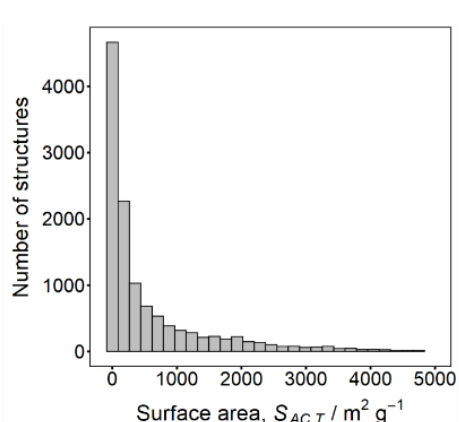

Surface area, $S_{A C, T} / \mathrm{m}^{2} \mathrm{~g}^{-1}$

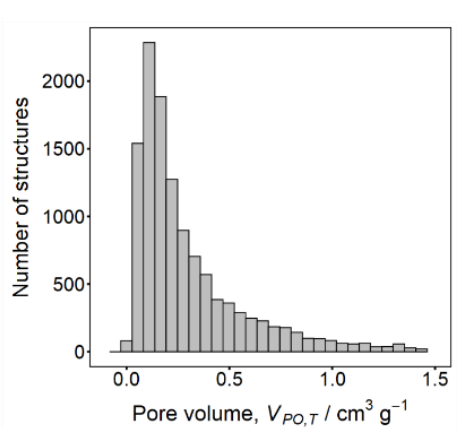

b.
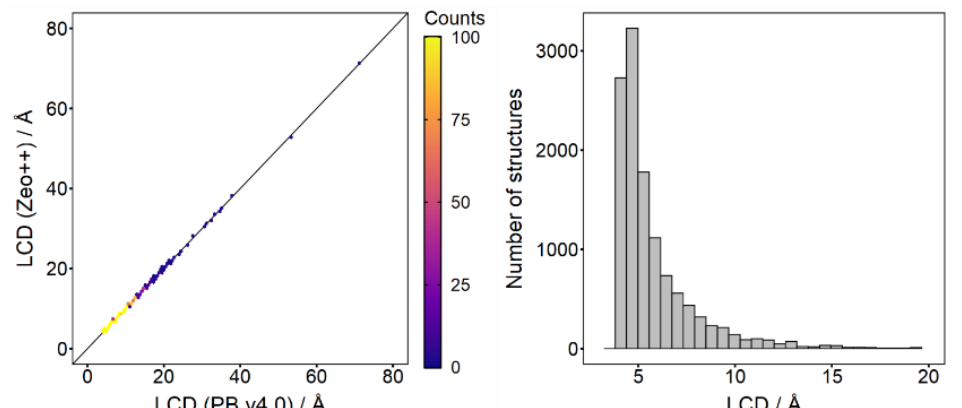

d.
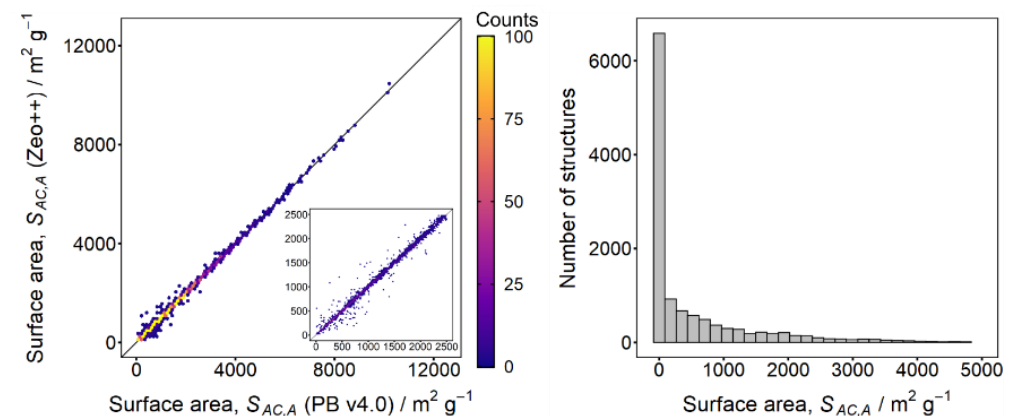

f.

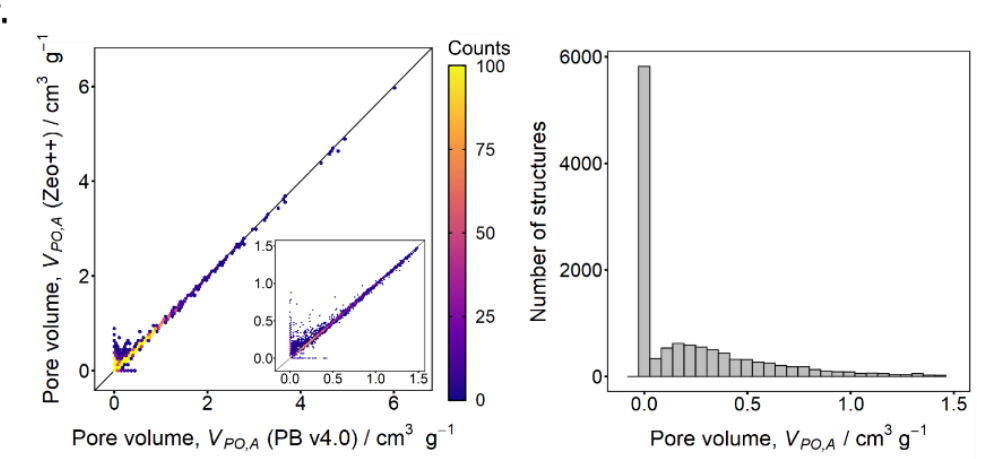

Figure 7. Comparison between results obtained from Zeo++ and PB v4.0. a. The pore limiting diameter, PLD; b. largest cavity diameter, LCD; c. total surface area, $S_{A C, T}$; d. network-accessible surface area, $S_{A C, A}$; e. total probe-occupiable volume, $V_{P O, T} ;$ f. network-accessible probe-occupiable volume and $V_{P O, A}$. Left panels show the parity plots, right panels show data distribution within 12,052 MOFs. 
We now turn our attention to the comparison of the pore volumes obtained using the three codes. Specifically, we focus our discussion on the network-accessible probe-occupiable $V_{P O, A}$ and the total probe-occupiable volume $V_{P O, T}$. In the case of the total probe-occupiable volume, PB v4.0 and Zeo++ are in a reasonable agreement with each other. At the same time, for the network-accessible probeoccupiable volume, we see again more scattering, particularly in the region of very dense materials with very small pore volumes (Fig. 7e-f). When looking at the the data distribution within the set of 12,052 porous MOFs, it is clear that the vast majority of structures is very microporous. In fact, more than 7,000 structures out of 12,052 have $V_{P O, T}$ below $0.25 \mathrm{~cm}^{3} / \mathrm{g}$. In the case of the network-accessible volume, $V_{P O, A}$, we notice a large number of structures having near-zero values. This subset of materials contains some materials with very low porosity and surface areas, in general, but also some materials, with appreciable total pore volume and surface area, but with PLDs smaller than the size of the probe nitrogen particle. These materials can be promising candidates for kinetic gas separations, based on the fine differences in sizes of the diffusing molecules.

In the case of RASPA, we can also obtain the helium pore volume and, therefore, we also explore this property using the helium volume fraction $F_{H e, T}=V_{H e, T} / V$ (for a more convenient representation). Figure 8 shows the parity graphs. There is a very good agreement between PB v4.0 and RASPA, as this property is calculated consistently between these two codes. As has been already discussed, in our definition and in the case of Zeo++, this property corresponds to the helium probecenter volume. Not surprisingly, there is a significant amount of scattering in the parity graph between PB v4.0 and Zeo++ (Fig. 8, right). In their study, Ongari et al. outlined several scenarios under which Eq. 7 underestimates, overestimates and agrees with the geometric pore volume and we refer the reader to that publication ${ }^{29}$.
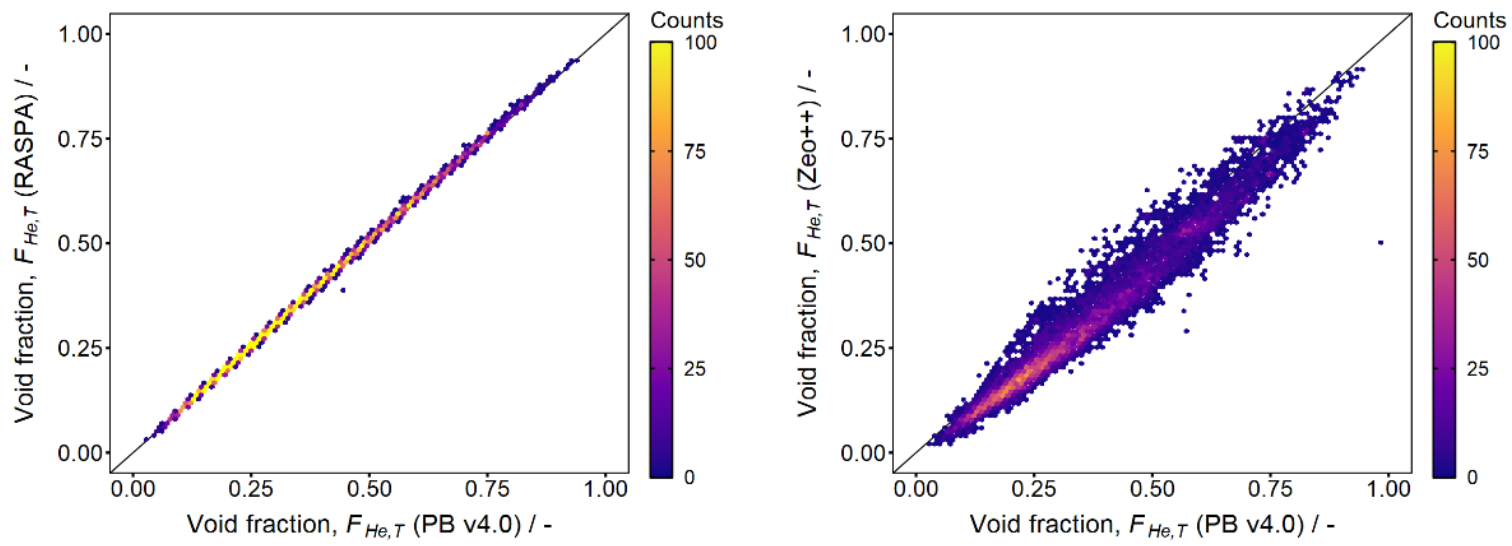

Figure 8. Parity graphs for helium pore volume fraction $\boldsymbol{F}_{\boldsymbol{H} \boldsymbol{e}, \boldsymbol{T}}$. Resultsfor PB v4.0 and RASPA are shown on the left; and for PB v4.0 and Zeo++ on the right.

\subsubsection{Error analysis}

We then moved to study the error analysis for the different textural parameters analyzed, i.e. the total and network-accessible probe-occupiable volume, $V_{P O, T}$ and $V_{P O, A}$, the total and network-accessible surface area, $S_{A C, T}$ and $S_{A C, A}$, and the pore limiting diameter, PLD. Here, the relative error is deifned

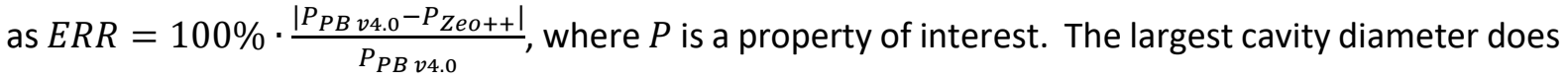


not feature materials for which predictions from PB v4.0 and Zeo++ deviate by more than $10 \%$.. Figure 9 shows the parity plots, correlations and error distributions for the different parameters. For for $V_{P O, T}$, there are have 4,824 (40.03\%) MOFs with an error exceeding $10 \%$ and with values from PB v4.0 systematically exceeding those from Zeo++ (Figure 9, first row). It is also clear that the vast majority of these MOFs correspond to low pore volumes (below $0.25 \mathrm{~cm}^{3} / \mathrm{g}$ ). For the network-accessible probeoccupiable volume $V_{P O, A}$, we observe 986 (8.18\%) MOFs with an error exceeding $10 \%$ (Figure 9, second row). Out of them, 574 MOFs have $V_{P O, A}$ values from PB v4.0 larger than Zeo++. The errors occur predominantly for MOFs with $V_{P O, A}$ values in the range $0-0.75 \mathrm{~cm}^{3} / \mathrm{g}$; errors exceeding $25 \%$ occur only for materials with $V_{P O, A}$ values around $0-0.5 \mathrm{~cm}^{3} / \mathrm{g}$. Several structures (ca. 100) have $100 \%$ error: in this case, the structure is considered non-accessible in Zeo++, but accessible on PB v4.0. Also, a small number of structures has errors much higher than $100 \%$ (e.g. 1000\%): these are the cases where, according to PB v4.0, the MOFs have very low $V_{P O, A}$; however, these structures are deemed more accessible and therefore with much larger $V_{P O, A}$ in Zeo++.

In the case of the total surface area, $S_{A C, T}$, we have a total number of 2,261 (18.76\%) MOFs with errors exceeding $10 \%$ (Figure 9, $3^{\text {rd }}$ row); importantly, the maximum values found for MOFs having these discrepancies are below $500 \mathrm{~m}^{2} / \mathrm{g}$. Out of the 2,261 MOFs, 1,488 show Zeo++ $S_{A C, T}$ values larger than PB v4.0. Similar to the previous properties, MOFs with significant errors are concentrated in the low surface area regime: errors exceeding $100 \%$ correspond to the materials with surface areas below $5 \mathrm{~m}^{2} / \mathrm{g}$. When looking at the network-accessible surface area, $S_{A C, A}$, we found 2,396 MOFs (19.88\%) with errors exceeding $10 \%$ (Figure $9,4^{\text {th }}$ row). 2,210 MOFs show an error of $100 \%$; these MOFs have zero network-accessible surface areas according to Zeo++ but non-zero values in PV v4.0 - this is observed as the straight horizontal line of points (Figure $9,4^{\text {th }}$ row, left). Seven MOFs also feature zero values of this property according to PB v4.0 and non-zero values in Zeo++ in the range of $90-600 \mathrm{~m}^{2} / \mathrm{g}$. We finally consider PLD, where there are 1,524 (10.40\%) MOFs with errors exceeding $10 \%$ (Figure 9 , $5^{\text {th }}$ row). Within this group, Zeo++ systematically predicts higher values. We also note that the majority of these MOFs are grouped around very low values of the PLD (below $2.5 \AA$ ). According to the error distribution, from 1,524 MOFs with an error exceeding 10\%, 1,142 show an error between 10 and 20\% and 264 between 20 and $30 \%$. 

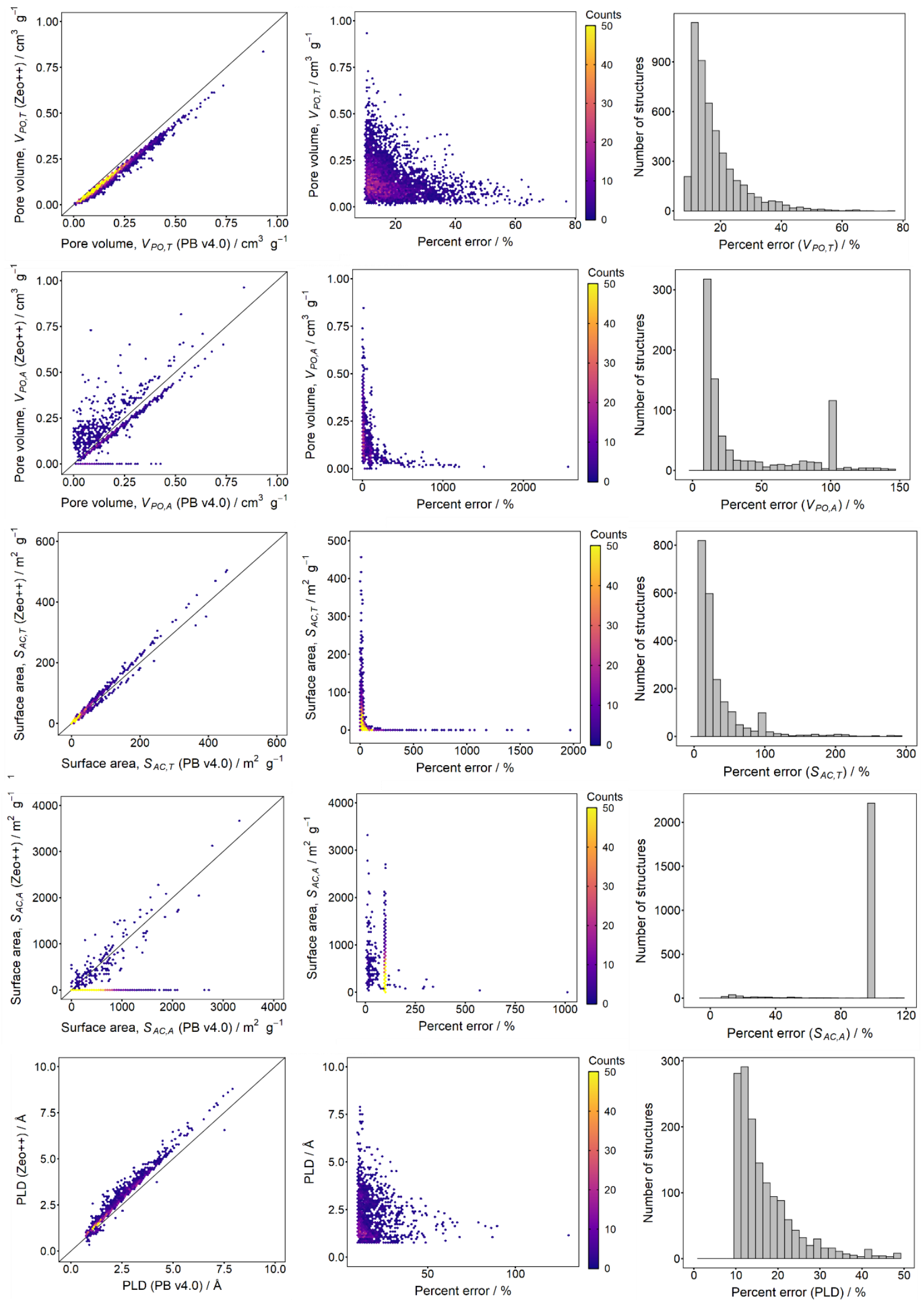

Figure 9. Error analysis of the structural properties. From top down: the total and network-accessible probeoccupiable volume, $V_{P O, T}$ and $V_{P O, A}$, the total and network-accessible surface area, $S_{A C, T}$ and $S_{A C, A}$, and the pore limiting diameter, PLD. Parity plots of the 4,824 structures with the error exceeding $10 \%$ (left), the correlation 
between the different properties and the magnitude of the error (center), and error distribution for each property.

From the results presented above in the comparison of PB v4.0 and Zeo++, it is clear that the total properties (i.e. volume and surface area) exhibit relatively limited scattering in the parity plots - the main differences between both codes are observed, predominantly, for those materials that feature low porosity. On the other hand, the network-accessible properties show higher scattering and therefore the reasons for the differences must be associated with how the network accessibility is obtained and to what factors this property is sensitive to. Let us outline three possible scenarios for when the results are expected to deviate significantly for PB v4.0 and Zeo++:

Scenario 1. This is associated with what probe molecules are used to assess the accessibility of the porous structure and to obtain the surface area. In PB v4.0, we effectively use two different probes: the accessibility is obtained using a nitrogen probe and the collision diameter value for its size; for the surface area we use nitrogen probe with the collision diameter multiplied by a factor 1.122 to account for the likely position of the adsorbed atoms at the distance of the energy minimum, rather than at the collision distance. To make Zeo++ calculation consistent with the PB v4.0, we invoked a setup, provided in the github depository, that is based on the two different probe sizes. This is not however the default protocol in Zeo++: in fact, it is recommended that the size of the probe used for the surface sampling is equal or smaller than the size of the probe employed for the accessibility analysis. In the majority of the cases, and as we observed for the results in Table 7, it does not present a problem, giving consistent results between the two programs. However, it is a problem for the MOFs where the PLD is very close to $3.72 \AA$. . In the case of PB v4.0, it treats these materials as nitrogen accessible. However, in Zeo++, these materials are not identified as accessible, leading to zero network-accessible surface area. This is the reason for the string of 2,210 materials (flat line) with zero Zeo++ surface area and non-zero PB v4.0 surface area in Figure 9. To test this hypothesis, we calculated the PLD distribution for this set of materials (see ESI, section S4), showing that, indeed, all these materials feature PLDs very close to $4 \AA$, where we expect the two codes to become sensitive to the details of how connectivity in the porous space is calculated.

Scenario 2. The second scenario is similar to the first one. If compartments of the porous space are separated by very narrow windows leading to side pores, comparable in size to the probe particle, the resulting properties become sensitive to sizes of the probes and the algorithms employed to assess accessibility. If PB v4.0 "sees" these side pores and Zeo++ does not, it would lead to the values of the accessible properties being higher in PB v4.0 compared to Zeo++ and vice-versa.

For both scenarios 1 and 2, we need to be aware that Zeo++ and PB v4.0 use different algorithms to assess percolation of the porous space across the periodic boundaries, and this may also be the source of disagreement.

Scenario 3. Scenario 3 is a rather general shortcoming of the lattice representation of the porous space: as the pores become smaller, and hence the surface area and porosity, their values become more sensitive to the resolution of the lattice grid.

\subsubsection{Analysis of a reduced set of MOFs with practical porosity and surface area values}

Not all porous materials are useful for adsorption applications. Typically, for gas storage, we are interested in high pore volume and high surface area materials. Surface areas of typical industrial adsorbents are in the hundreds of $\mathrm{m}^{2} / \mathrm{g}$, whereas pore volumes of most of MOFs and zeolites exceed $0.25 \mathrm{~cm}^{3} / \mathrm{g}$ (for zeolites, see First et al. ${ }^{15}$ ). Specifically, within the considered set of $12,052 \mathrm{MOFs}, 3,598$ 
MOFs have a total surface area below $50 \mathrm{~m}^{2} / \mathrm{g}, 7,094 \mathrm{MOFs}$ have a total pore volume below $0.25 \mathrm{~cm}^{3} / \mathrm{g}$ and 7,252 MOFs have low surface area or/and porosity according to the criteria above. In section, we exclude these MOFs from consideration and focus on the remaining structures.

Within this reduced set of MOFs (ca. 4,800 structures) the errors (i.e. the differences in structural properties from PB v4.0 and Zeo++) can be summarized as follows. For the total pore volume, 542 structures have outliers with errors larger than $10 \%$; most of these errors are around $10-20 \%$. For the network-accessible volume, 449 structures have errors larger than $10 \%$. These subgroups can be separated into two subcategories: those that have errors within $25 \%$ and those with around $100 \%$. The latter category is associated with one of the codes considering the structure as accessible and highly porous, whereas the other code does not consider it as accessible. For the total surface area within the reduced dataset, only 70 structures have an error larger than $10 \%$, and most of the structures are with 10-15\% error. For the network-accessible surface area, 1,122 structures show errors larger than $10 \%$. Within this group, 995 structures show errors of $100 \%$ and 993 are structures with a non-zero value for PB v4.0 and zero value for Zeo++. The rest of the structures show error values between $10-30 \%$. The complete set of figures and error analysis for this reduced set is provided in the ESI file, sections S5 and S6.

In our analysis, by removing low porosity systems, we have eliminated the potential errors associated with Scenario 3. The remaining disagreement between PB v4.0 and Zeo++ must be now strictly associated with percolation and network accessibility analysis. While the actual detection of the difference would require a more detailed analysis of the individual structures, a reasonable initial support for this hypothesis would be provided by the analysis of the PLD in the outlier MOFs. Indeed, Figure S10 in the ESI shows the distribution of the PLD for the set of 449 MOFs that show $>10 \%$ deviation in the network-accessible pore volume. This figure clearly demonstrates that the vast majority of these materials feature PLDs below $4 \AA$. This is the regime where we expect higher sensitivity in the percolation algorithms, according to Scenarios 1 and 2.

\subsection{Material informatics with PB v4.0 and MOF/PCA Explorer}

In section 3.2 we assembled a database of geometric properties of materials within the experimental CSD MOF subset and explored the prediction of geometric properties from several available computational tools. What type of questions can we ask using this data? In principle, one would hope to use this data to explore current limits in the geometrical properties of materials, correlations between various properties and clustering of the properties together, and to engineer advanced features to be explored in the Machine Learning algorithms ${ }^{43}$. This, on its own, may guide the design of new materials or help with the search for a material with particular structural characteristics, such as a given PLD for kinetic separations. Combined with the functional properties of the materials, such as the adsorption characteristics, this forms a platform for material discovery and process optimization. An example of such a discovery platform is provided at the Materials $\mathrm{Cloud}^{44}$.

A discovery platform requires material informatics tools. The data we deal with is intrinsically multidimensional, and therefore the tools required to reveal the trends and correlations within the data generally aim to reduce the dimensionality of the space. Based on our previous work ${ }^{23-25}$, we have developed two sets of tools available for interactive, dynamic exploration: the Metal-Organic Framework Data Visualisation tool (https://aaml-explorer-geo-prop.herokuapp.com) and the Principal Component Analysis Data Visualisation tool (https://aaml-pca-geo-prop.herokuapp.com), 
which can be used without any prior programming knowledge. The MOF explorer allows the user to filter the data according to a selection of various criteria, such as the values of the selected geometric properties within certain intervals. It also visualizes and animates the data using 2D and 3D plots. The data on these plots can be further augmented using color and the size of the symbols, expanding the number of properties that can be simultaneously visualized to 5 . Finally, it can provide statistics on the distribution of properties within the dataset. The second tool, the Principle Component Analysis (PCA) Explorer, allows one to explore of the data set through feature correlation analysis and PCA obtaining biplots, loading plots, squared cosine and contribution plots. Using these tools, we have explored some of the features of the CSD MOF subset obtained with PB v4.0.

Figure 10 shows an example of representing 4-dimensional space of values: pore volume, surface area, PLD and LCD/PLD ratio using 2D plots. One particular question one may ask using this analysis is the nature and properties of the materials sitting on the edge of the cloud of points shown in Figure 10. The MOFs on the top right of the plot corresponds to materials with $1 D$ or $2 D$ framework dimensionalities; this is, metal-organic chains or sheets, respectively. For now, we do not consider them, as they are more unlikely to find practical adsorption applications. We labeled other structures with 3D pores on the edge (or close to) of the cloud in Figure 10 and provided their data in Table 4.

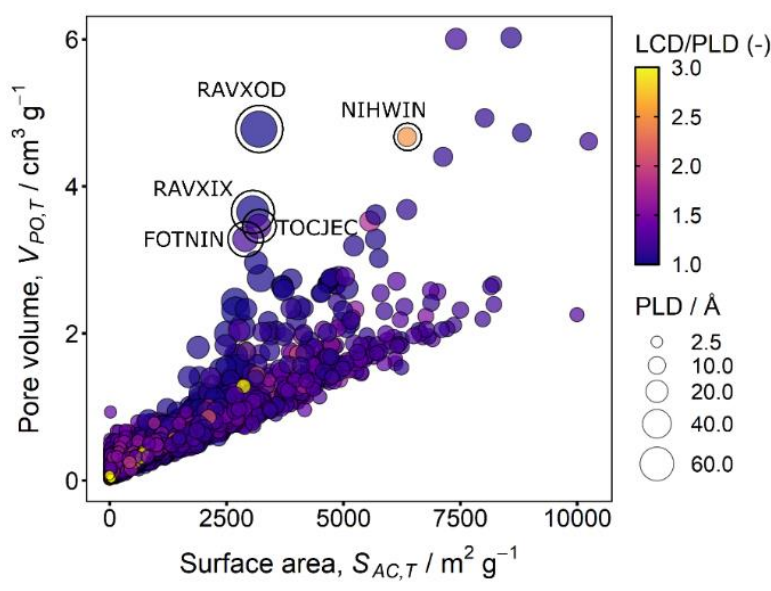

Figure 10. 4-dimensional analysis in a 2D plot. Total probe-occupiable pore volume $V_{P O, T}$ vs. total accessible surface area $S_{A C, T}$. Color bar indicates the value of the LCD/PLD ratio and the size of the circles indicates the value of the PLD.

Table 4. Materials close to the edge of the cloud of properties in Figure 14 and their properties. Showing the CSD refcode, density, total helium volume $\left(F_{H e, T}\right)$ fraction, total surface area $\left(S_{A C, T}\right)$, total probe-occupiable volume $\left(V_{P O, T}\right)$, por limiting diameter (PLD), largest cavity diameter (LCD), LCD/PLD ratio and pore dimensionality.

\begin{tabular}{|c|c|c|c|c|c|c|c|c|}
\hline Refcode & $\begin{array}{l}\text { Density } \\
\left(\mathrm{g} / \mathrm{cm}^{3}\right)\end{array}$ & $F_{H e, T}$ & $\begin{array}{c}S_{A C, T} \\
\left(\mathrm{~m}^{2} / \mathrm{g}\right)\end{array}$ & $\begin{array}{c}V_{P O, T} \\
\left(\mathrm{~cm}^{3} / \mathrm{g}\right)\end{array}$ & $\begin{array}{l}\text { PLD } \\
\text { (Å) }\end{array}$ & $\begin{array}{l}\text { LCD } \\
(\AA ̊)\end{array}$ & LCD/PLD & Porosity \\
\hline NIHWIN & 0.193 & 0.929 & 6358.6 & 4.671 & 12.98 & 32.34 & 2.49 & $3 D$ \\
\hline RAVXOD & 0.179 & 0.875 & 3190.2 & 4.778 & 71.08 & 71.20 & 1.0 & $1 \mathrm{D}$ \\
\hline RAVXIX & 0.228 & 0.864 & 3061.5 & 3.654 & 52.79 & 53.13 & 1.0 & $1 \mathrm{D}$ \\
\hline TOCJEC & 0.253 & 0.900 & 3187.1 & 3.457 & 25.57 & 30.94 & 1.21 & $3 D$ \\
\hline FOTNIN & 0.270 & 0.900 & 2901.1 & 3.284 & 28.18 & 33.41 & 1.18 & $2 D$ \\
\hline
\end{tabular}


What are these materials listed in Table 4? As the CSD MOF subset reports experimental structures, the reference codes in Figure 10 and Table 4 correspond to the actual synthesized and reported materials. RAVXOD and RAVXIX are IRMOF-74-IX and -XI, respectively ${ }^{45}$. These MOFs belong to a series of MOFs isoreticular to CPO-27/MOF-74 (eth topology) and are made of Mg-clusters and derivatives of dioxidoterephathalate with 9 (IRMOF-74-IX) or 11 (-XI) aromatic rings. NIWHIN is known as DUT-60 ${ }^{46}$. This MOF, isoreticular to DUT-6 (ith- $d$ topology) with elongated linkers, was designed in silico, its mechanical properties were studied computationally, and it was synthesized successfully. It is built from $\mathrm{Zn}_{4} \mathrm{O}\left(\mathrm{CO}_{2}\right)_{6}$ clusters connected by bbc ${ }^{3-}$ and bcpbd ${ }^{2-}$ linkers, generating a pore system with large mesopores surrounded by 8 smaller mesopores. TOCJEC is bio-MOF- $2^{47}$. This MOF was synthesized from bio-MOF-100, a Zn-based MOF with mesoporous interconnected channels ${ }^{48}$. For the synthesis of bio-MOF-2, they followed a stepwise ligand exchanged strategy, replacing the shorter ligands of bio-MOF-100 with longer ligands to transform the crystal in a new one with the same topology and bigger pores. FOTNIN is PCN- $777^{49}$. This zirconium MOF is a highly stable MOF with $\beta$ cristobalite topology and big cages.

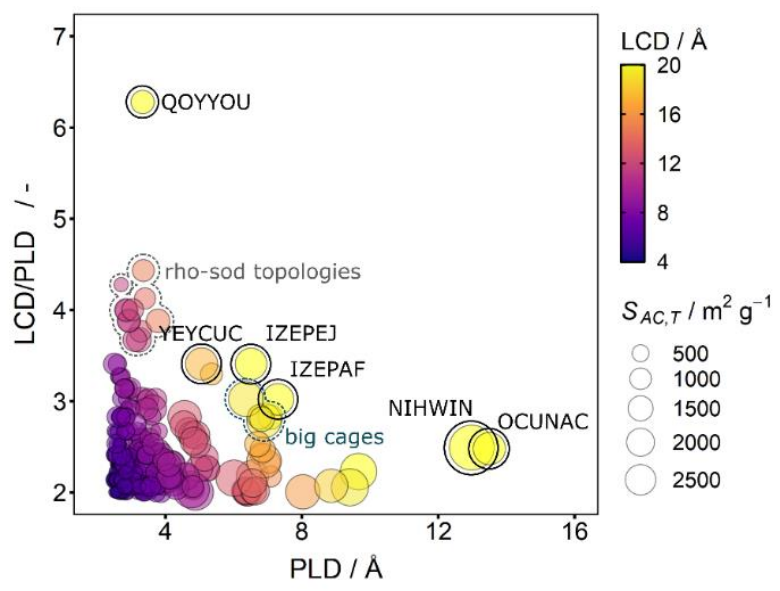

Figure 11. 4-dimensional analysis in 2D. LCD/PLD ratio vs PLD, $\AA$. Color bar indicates the value of LCD and size of the point indicates the value of the total accessible surface area, $S_{A C, T}$.

In the ESI, section S8, we provide PCA of the geometric properties of MOFs. Interestingly, the ratio of the LCD/PLD emerged as a property not strongly correlated with other properties under consideration. The PCA showed that three principal components can explain $90.27 \%$ of the variance within the dataset, and these three principal components are highly correlated with the ratio of the $\mathrm{LCD} / \mathrm{PLD}$, the total pore volume $\left(V_{P O, T}\right)$ and the density. Therefore, although the geometrical properties calculated with PB v4.0 are highly correlated, these three mostly independent properties can widely explain the distribution of the data.

From the application point of few, we were curious whether we could identify materials with very large values of $L C D / P L D$, as large pores connected by narrow windows could be promising materials for kinetic separations, switch-on/switch-off or stimuli-responsive gas storage applications. For most of the MOFs, the LCD/PLD ratio sits around a value of 1.5 and this corresponds to slightly different pore diameters within the same channel. When the value of this property increases, we will find 
structures with very small PLDs. Many MOFs are showing large values for LCD/PLD that are worth exploring in more detail, such NIWHIN, already mentioned in Figure 10 and Table 4.

Figure 11 shows the LCD/PLD ratio as a function of PLD, with the color bar and the size of the circles giving information about the LCD and the total accessible surface area, respectively. The structures that clearly sit at the edge of the distribution are QOYYOU, with very high ratio LCD/PLD and relatively low PLD, NIHWIN (DUT-60) and OCUNAC (known as MIL-101), that show the opposite trend (large PLD, small LCD/PLD ratio). In the range of medium values, YEYCUC (PCN-58), IZEPAF (MIL-hypo-1) and IZEPAF (MIL-hypo-2) show both large PLDs and LCD/PLD ratios as well as high surface areas. In addition, there are two groups of structures near these ones. The first group, highlighted in grey, show high LCD/PLD value but small PLD and correspond to structures with rho or sod type of topologies. These topologies have big cages with narrow windows connecting them. The second group, highlighted in blue, shows a large PLD and LCDs three times higher than the previous group; REWNEO (NU-125) and GUPDEB (CdIF-9) belong here. Table 5 shows the geometrical properties of these MOFs, except for NIHWIN, which was already reported above.

Table 5. Materials close to the edge of the cloud of properties in Figure 11 and their properties. Showing the CSD refcode, density, total helium volume $\left(F_{H e, T}\right)$ fraction, total surface area $\left(S_{A C, T}\right)$, Total probe-occupiable volume $\left(V_{P O, T}\right)$, pore limiting diameter $(P L D)$, largest cavity diameter (LCD), LCD/PLD ratio and pore dimensionality.

\begin{tabular}{|c|c|c|c|c|c|c|c|c|}
\hline Refcode & $\begin{array}{l}\text { Density } \\
\left(\mathrm{g} / \mathrm{cm}^{3}\right)\end{array}$ & $F_{H e, T}$ & $\begin{array}{c}S_{A C, T} \\
\left(\mathrm{~m}^{2} / \mathrm{g}\right)\end{array}$ & $\begin{array}{c}V_{P O, T} \\
\left(\mathrm{~cm}^{3} / \mathrm{g}\right)\end{array}$ & $\begin{array}{l}\text { PLD } \\
(\AA ̊)\end{array}$ & $\begin{array}{l}\text { LCD } \\
(\AA \AA)\end{array}$ & LCD/PLD & Porosity \\
\hline QOYYOU & 0.945 & 0.701 & 1247.0 & 0.642 & 3.31 & 20.93 & 6.32 & $3 D$ \\
\hline OCUNAC & 0.450 & 0.803 & 2893.0 & 1.762 & 13.50 & 33.48 & 2.48 & $3 D$ \\
\hline IZEPEJ & 0.585 & 0.763 & 2741.4 & 1.253 & 6.51 & 22.19 & 3.41 & $3 D$ \\
\hline IZEPAF & 0.612 & 0.755 & 2727.2 & 1.173 & 7.30 & 22.05 & 3.02 & $3 D$ \\
\hline YEYCUC & 0.554 & 0.768 & 2862.7 & 1.282 & 5.06 & 17.25 & 3.41 & $3 D$ \\
\hline REWNEO & 0.578 & 0.833 & 3483.8 & 1.308 & 6.36 & 19.23 & 3.02 & $2 \mathrm{D}$ \\
\hline GUPDEB & 0.945 & 0.644 & 1448.7 & 0.635 & 7.07 & 19.98 & 2.82 & $3 D$ \\
\hline
\end{tabular}

Again, the CSD reference codes in Table 5 correspond to real structures. QOYYOU is a structure designed computationally and synthesized successfully by Bai et al. based on $\mathrm{Zn}_{4} \mathrm{O}-7$ clusters ${ }^{50}$. Its pore system comprises three types of cages with sizes ranging from the micro to the mesoporous scales. The largest cage is connected to another 6 large-size cages, 8 medium-size cages and 12 small-size cages. OCUNAC is MIL-101 from Ferey and co-workers, which was "tailor-made" by assembling its building blocks using computational strategies ${ }^{51}$. The synthesized framework is characterized by two types of mesoporous cages with pentagonal and hexagonal windows. YEYCUC is PCN-58 from Jiang and co-workers ${ }^{52}$. This MOF belongs to an isoreticular series of MOFs similar to UiO-type, Zr-based MOFs, and was designed with appended azide groups to control post-synthetic functionalization. The resulting MOF shows two polyhedral microporous cages: one tetrahedral and one octahedral. IZEPAF and IZEPEJ are MIL-hypo-1 and -2, two hypothetical MOFs designed computationally by MellotDraznieks and co-workers ${ }^{53}$. IZEPAF has three types of cages, cubic, spherical and rhombicuboactahedral in the range of micro- and mesopores. IZEPEJ has also three types of cages: tetrahedral, cubic and a spherical one constructed by the combination of the other two. REWNEO is NU-125 by Wilmer et al. ${ }^{54}$, a MOF with rht topology, Cu-Cu paddle-wheels as metal clusters and 
characterized by four different types of cages with sizes ranging from $11 \AA$ to $24 \AA$. GUPDEB is CdIF-9 by Tian et al. ${ }^{55}$, a MOF with rho topology that belongs to a group of cadmium imidazolate frameworks, known as CdIFs, which are regarded as more open than their Zn- or Co-based analogs. In section S9 of the ESI we provide computer visualizations of the structures within Table 5.

We emphasize here that the main objective of this section was not to identify the best MOF for a specific application, but simply to illustrate how the available tools can be used to reduce the dimensionality of the space of MOF geometric properties and to reveal some interesting, hidden correlations between them. We encourage the reader to explore these tools, by using their own data and their own derived features.

\section{Outlook}

The objective of this paper was to introduce the new version of the PoreBlazer code for structural characterization of porous materials. We provided a comprehensive review of the geometric properties that can be obtained for crystal structure given coordinates of its atoms, the algorithms behind the calculation of these properties, and elaborated on the links between the geometric properties of porous materials and the properties that can be actually measured experimentally.

There are now several codes available to obtain these geometric properties, differing in the algorithms employed, efficiency, availability of the source code, platforms etc. In general, having several alternative codes for the same computational task it generally a very positive thing scientific advancement. For example, healthy competition between several coexisting molecular dynamic codes (LAMMS, GROMOS etc.) has been driving efforts to improve performance of the codes, to provide accurate and comprehensive documentation, and ultimately, to provide alternative platforms to validate the algorithms and to ensure reproducibility of the data. Sarkisov group has recently made the first step towards similar transparency and cross-validation in the domain of the Monte Carlo codes, particularly in application to the adsorption problems.

To address this issue here we provided comprehensive comparison of the properties obtained from the currently available codes, PB v4.0, Zeo++ and RASPA. While writing this article we became aware of another code PorosityPlus ${ }^{56}$, however we have not tested its capabilities here. Overall, the codes are predominantly consistent with each other, which is definitely reassuring. The most sensitive properties that exhibit a significant degree of scattering are the properties that require analysis of the accessibility of different region of the porous structure. This scattering is exacerbated where structures have low porosity and surface area or/and feature channels of sizes comparable to the size of the probe used to explore connectivity of the porous space. Identifying what properties are sensitive to the algorithms employed is also an important outcome of the study.

This article should not be considered as a promotion of a specific code - likely the codes will find different scopes and niches complementing each other. The significant advantage of Zeo++ is the fact that it works with small asymmetric unit cells and cif files, leading to a substantial computational efficiency (see a more complete analysis in the SI). However, for the expanded unit cell such as presented in the section 3.1, PB v4.0 seems to be more efficient (this is without yet taking any advantage of the lattice structure of the code, such GPU and parallelization using MPI and OpenMP. This suggests that the possible area of PB v4.0 application is the disordered materials (model activated carbons, MOFs with defects, polymers) based on unit cells of significant size to operate on the length 
scales compatible with the disorder features. We further note that the fact that PB v4.0 uses lattice representation and effectively pre-calculates the distances between possible location of the adsorbate molecules and atoms of the adsorbent structure, allows PB v4.0 to go beyond purely geometric features and explore properties that now depend on the intermolecular interactions. An obvious example of this is the helium volume fraction obtained by default in PB v4.0. However, with the interaction parameters changed to the spherical molecule of interest (e.g. methane, noble gases), the same simulation can be used to obtain the Henry's constants of adsorption (Eq. 9). Lattice representation of the porous space then opens an opportunity to explore adsorption in Henry's regime and free energy landscapes within the porous materials ${ }^{57-59}$.

Using PB v4.0, Zeo++ and RASPA we obtained geometric properties of ca. 12,000 porous MOFs within CDS MOF database and made the data available on the github depository. The analysis of this data indicates that within this set of materials still a very significant proportion (close to 60\%) of MOFs have very low porosity and surface area and are unlikely candidates for any adsorptive applications. This is in contrast to the hypothetical MOF and ZIF databases featuring hundreds of thousands of structures: it seems only a small fraction of this proper porous MOF universe has been realized experimentally and it is an interesting philosophical question on why it is so.

The data is available online and it opens an opportunity for readers explore this data and mine it for some interesting correlations. Here, we presented a case study where visualization of the multidimensional data, combined with some statistical analysis such as PCA, was used to efficiently discover MOFs with interesting characteristics (e.g. large LPD/PLD ratio values). MOF databases have been now assembled by several groups for both real and hypothetical structures and it is an ongoing scientific quest to understand what kind of scientific questions we can pose to these databases. For example, a recent study by Moosavi et al. ${ }^{60}$, asked whether the possible parameter space of MOFs is uniformly sampled and what clusters form within the current universe of MOFs. We believe, however, we are just at the beginning of the realization of the full potential of the material informatics tools in discovery of new materials and new applications.

\section{Acknowledgements}

LS would like to thank Prof. Shane Telfer of Massey University and his group for early tests of the code, Drs. Senja Barthel and Deniele Ongari for helpful comments and clarifications; and many scientists around the world who provided feedback and pointed out to the bugs in the code.

\section{References}

1. Furukawa, H.; Cordova, K. E.; O'Keeffe, M.; Yaghi, O. M., The chemistry and applications of metal-organic frameworks. Science 2013, 341 (6149), 1230444.

2. Pimentel, B. R.; Parulkar, A.; Zhou, E. K.; Brunelli, N. A.; Lively, R. P., Zeolitic imidazolate frameworks: next-generation materials for energy-efficient gas separations. ChemSusChem 2014, 7 (12), 3202-40.

3. Ding, S. Y.; Wang, W., Covalent organic frameworks (COFs): from design to applications. Chem Soc Rev 2013, 42 (2), 548-68.

4. Düren, T.; Sarkisov, L.; Yaghi, O. M.; Snurr, R. Q., Design of New Materials for Methane Storage. Langmuir 2004, 20 (7), 2683-2689.

5. Wilmer, C. E.; Leaf, M.; Lee, C. Y.; Farha, O. K.; Hauser, B. G.; Hupp, J. T.; Snurr, R. Q., Large-scale screening of hypothetical metal-organic frameworks. Nature Chemistry 2012, 4 (2), 8389. 
6. Colón, Y. J.; Snurr, R. Q., High-throughput computational screening of metal-organic frameworks. Chemical Society Reviews 2014, 43 (16), 5735-5749.

7. Düren, T.; Millange, F.; Férey, G.; Walton, K. S.; Snurr, R. Q., Calculating Geometric Surface Areas as a Characterization Tool for Metal-Organic Frameworks. The Journal of Physical Chemistry $C$ 2007, 111 (42), 15350-15356.

8. Figueroa-Gerstenmaier, S.; Avalos, J. B.; Gelb, L. D.; Gubbins, K. E.; Vega, L. F., Pore size distribution of porous glasses: A test of the independent pore model. Langmuir 2003, 19 (20), 85928604.

9. Gelb, L. D.; Gubbins, K. E., Characterization of porous glasses: Simulation models, adsorption isotherms, and the Brunauer-Emmett-Teller analysis method. Langmuir 1998, 14 (8), 2097-2111.

10. Gelb, L. D.; Gubbins, K. E., Pore size distributions in porous glasses: A computer simulation study. Langmuir 1999, 15 (2), 305-308.

11. Winterfeld, P. H.; Scriven, L. E.; Davis, H. T., Percolation and conductivity of random twodimensional composites. Journal of Physics C: Solid State Physics 1981, 14 (17), 2361-2376.

12. Sarkisov, L.; Harrison, A., Computational structure characterisation tools in application to ordered and disordered porous materials. Molecular Simulation 2011, 37 (15), 1248-1257.

13. Sarkisov, L.; Kim, J., Computational structure characterization tools for the era of material informatics. Chemical Engineering Science 2015, 121, 322-330.

14. First, E. L.; Floudas, C. A., MOFomics: Computational pore characterization of metal-organic frameworks. Microporous and Mesoporous Materials 2013, 165, 32-39.

15. First, E. L.; Gounaris, C. E.; Wei, J.; Floudas, C. A., Computational characterization of zeolite porous networks: an automated approach. Physical Chemistry Chemical Physics 2011, 13 (38), 17339-17358.

16. Pinheiro, M.; Martin, R. L.; Rycroft, C. H.; Haranczyk, M., High accuracy geometric analysis of crystalline porous materials. CrystEngComm 2013, 15 (37), 7531-7538.

17. Pinheiro, M.; Martin, R. L.; Rycroft, C. H.; Jones, A.; Iglesia, E.; Haranczyk, M., Characterization and comparison of pore landscapes in crystalline porous materials. Journal of Molecular Graphics and Modelling 2013, 44, 208-219.

18. Willems, T. F.; Rycroft, C. H.; Kazi, M.; Meza, J. C.; Haranczyk, M., Algorithms and tools for high-throughput geometry-based analysis of crystalline porous materials. Microporous and Mesoporous Materials 2012, 149 (1), 134-141.

19. Foster, M. D.; Rivin, I.; Treacy, M. M. J.; Delgado Friedrichs, O., A geometric solution to the largest-free-sphere problem in zeolite frameworks. Microporous and Mesoporous Materials 2006, 90 (1), 32-38.

20. Dubbeldam, D.; Calero, S.; Ellis, D. E.; Snurr, R. Q., RASPA: molecular simulation software for adsorption and diffusion in flexible nanoporous materials. Molecular Simulation 2016, 42 (2), 81 101.

21. Coudert, F.-X., Reproducible Research in Computational Chemistry of Materials. Chemistry of Materials 2017, 29 (7), 2615-2617.

22. Moghadam, P. Z.; Li, A.; Wiggin, S. B.; Tao, A.; Maloney, A. G. P.; Wood, P. A.; Ward, S. C.; Fairen-Jimenez, D., Development of a Cambridge Structural Database Subset: A Collection of MetalOrganic Frameworks for Past, Present, and Future. Chemistry of Materials 2017, 29 (7), 2618-2625.

23. Moghadam, P. Z.; Islamoglu, T.; Goswami, S.; Exley, J.; Fantham, M.; Kaminski, C. F.; Snurr, R. Q.; Farha, O. K.; Fairen-Jimenez, D., Computer-aided discovery of a metal-organic framework with superior oxygen uptake. Nature Communications 2018, 9 (1), 1378.

24. Matito-Martos, I.; Moghadam, P. Z.; Li, A.; Colombo, V.; Navarro, J. A. R.; Calero, S.; Fairen-Jimenez, D., Discovery of an Optimal Porous Crystalline Material for the Capture of Chemical Warfare Agents. Chemistry of Materials 2018, 30 (14), 4571-4579.

25. Moghadam, P. Z.; Li, A.; Liu, X.-W.; Bueno-Perez, R.; Wang, S.-D.; Wiggin, S. B.; Wood, P. A.; Fairen-Jimenez, D., Targeted classification of metal-organic frameworks in the Cambridge structural database (CSD). Chemical Science 2020, 11 (32), 8373-8387. 
26. Walton, K. S.; Snurr, R. Q., Applicability of the BET Method for Determining Surface Areas of Microporous Metal-Organic Frameworks. Journal of the American Chemical Society 2007, 129 (27), 8552-8556.

27. Ambroz, F.; Macdonald, T. J.; Martis, V.; Parkin, I. P., Evaluation of the BET Theory for the Characterization of Meso and Microporous MOFs. Small Methods 2018, 2 (11), 1800173.

28. Gómez-Gualdrón, D. A.; Moghadam, P. Z.; Hupp, J. T.; Farha, O. K.; Snurr, R. Q., Application of Consistency Criteria To Calculate BET Areas of Micro- And Mesoporous Metal-Organic Frameworks. Journal of the American Chemical Society 2016, 138 (1), 215-224.

29. Ongari, D.; Boyd, P. G.; Barthel, S.; Witman, M.; Haranczyk, M.; Smit, B., Accurate Characterization of the Pore Volume in Microporous Crystalline Materials. Langmuir 2017, 33 (51), 14529-14538.

30. Myers, A. L.; Monson, P. A., Physical adsorption of gases: the case for absolute adsorption as the basis for thermodynamic analysis. Adsorption 2014, 20 (4), 591-622.

31. Brandani, S.; Mangano, E.; Sarkisov, L., Net, excess and absolute adsorption and adsorption of helium. Adsorption 2016, 22 (2), 261-276.

32. Neimark, A. V.; Ravikovitch, P. I., Calibration of Pore Volume in Adsorption Experiments and Theoretical Models. Langmuir 1997, 13 (19), 5148-5160.

33. Düren, T.; Bae, Y.-S.; Snurr, R. Q., Using molecular simulation to characterise metal-organic frameworks for adsorption applications. Chemical Society Reviews 2009, 38 (5), 1237-1247.

34. Yang, Q.; Liu, D.; Zhong, C.; Li, J.-R., Development of Computational Methodologies for Metal-Organic Frameworks and Their Application in Gas Separations. Chemical Reviews 2013, 113 (10), 8261-8323.

35. Dantas, S.; Neimark, A. V., Coupling Structural and Adsorption Properties of Metal-Organic Frameworks: From Pore Size Distribution to Pore Type Distribution. ACS Applied Materials \& Interfaces 2020, 12 (13), 15595-15605.

36. Landers, J.; Gor, G. Y.; Neimark, A. V., Density functional theory methods for characterization of porous materials. Colloids and Surfaces A: Physicochemical and Engineering Aspects 2013, 437, 3-32.

37. Cychosz, K. A.; Guillet-Nicolas, R.; García-Martínez, J.; Thommes, M., Recent advances in the textural characterization of hierarchically structured nanoporous materials. Chemical Society Reviews 2017, 46 (2), 389-414.

38. Dantas, S.; Sarkisov, L.; Neimark, A. V., Deciphering the Relations between Pore Structure and Adsorption Behavior in Metal-Organic Frameworks: Unexpected Lessons from Argon Adsorption on Copper-Benzene-1,3,5-tricarboxylate. Journal of the American Chemical Society 2019, 141 (21), 8397-8401.

39. Hoshen, J.; Kopelman, R., PERCOLATION AND CLUSTER DISTRIBUTION .1. CLUSTER MULTIPLE LABELING TECHNIQUE AND CRITICAL CONCENTRATION ALGORITHM. Physical Review B 1976, 14 (8), 3438-3445.

40. Humphrey, W.; Dalke, A.; Schulten, K., VMD: Visual molecular dynamics. Journal of Molecular Graphics 1996, 14 (1), 33-38.

41. Li, A.; Bueno-Perez, R.; Wiggin, S.; Fairen-Jimenez, D., Enabling efficient exploration of metal-organic frameworks in the Cambridge Structural Database. CrystEngComm 2020.

42. Fairen-Jimenez, D.; Moggach, S. A.; Wharmby, M. T.; Wright, P. A.; Parsons, S.; Düren, T., Opening the Gate: Framework Flexibility in ZIF-8 Explored by Experiments and Simulations. Journal of the American Chemical Society 2011, 133 (23), 8900-8902.

43. Jablonka, K. M.; Ongari, D.; Moosavi, S. M.; Smit, B., Big-Data Science in Porous Materials: Materials Genomics and Machine Learning. Chemical Reviews 2020.

44. Leopold Talirz, S. K., Elsa Passaro, Aliaksandr V. Yakutovich, Valeria Granata, Fernando Gargiulo, Marco Borelli, Martin Uhrin, Sebastiaan P. Huber, Spyros Zoupanos, Carl S. Adorf, Casper W. Andersen, Ole Schütt, Carlo A. Pignedoli, Daniele Passerone, Joost VandeVondele, Thomas C. 
Schulthess, Berend Smit, Giovanni Pizzi, Nicola Marzari, Materials Cloud, a platform for open computational science. 2020.

45. Deng, H.; Grunder, S.; Cordova, K. E.; Valente, C.; Furukawa, H.; Hmadeh, M.; Gándara, F.; Whalley, A. C.; Liu, Z.; Asahina, S.; Kazumori, H.; O’Keeffe, M.; Terasaki, O.; Stoddart, J. F.; Yaghi, O. M., Large-Pore Apertures in a Series of Metal-Organic Frameworks. Science 2012, 336 (6084), 1018-1023.

46. Hönicke, I. M.; Senkovska, I.; Bon, V.; Baburin, I. A.; Bönisch, N.; Raschke, S.; Evans, J. D.; Kaskel, S., Balancing Mechanical Stability and Ultrahigh Porosity in Crystalline Framework Materials. Angewandte Chemie International Edition 2018, 57 (42), 13780-13783.

47. Li, T.; Kozlowski, M. T.; Doud, E. A.; Blakely, M. N.; Rosi, N. L., Stepwise Ligand Exchange for the Preparation of a Family of Mesoporous MOFs. Journal of the American Chemical Society 2013, 135 (32), 11688-11691.

48. An, J.; Farha, O. K.; Hupp, J. T.; Pohl, E.; Yeh, J. I.; Rosi, N. L., Metal-adeninate vertices for the construction of an exceptionally porous metal-organic framework. Nature Communications 2012, 3 (1), 604.

49. $\quad$ Feng, D.; Wang, K.; Su, J.; Liu, T.-F.; Park, J.; Wei, Z.; Bosch, M.; Yakovenko, A.; Zou, X.; Zhou, H.-C., A Highly Stable Zeotype Mesoporous Zirconium Metal-Organic Framework with Ultralarge Pores. Angewandte Chemie International Edition 2015, 54 (1), 149-154.

50. Bai, S.; Zhang, W.; Ling, Y.; Yang, F.; Deng, M.; Chen, Z.; Weng, L.; Zhou, Y., Predicting and creating 7-connected $\mathrm{Zn} 4 \mathrm{O}$ vertices for the construction of an exceptional metal-organic framework with nanoscale cages. CrystEngComm 2015, 17 (9), 1923-1926.

51. Férey, G.; Mellot-Draznieks, C.; Serre, C.; Millange, F.; Dutour, J.; Surblé, S.; Margiolaki, I., A Chromium Terephthalate-Based Solid with Unusually Large Pore Volumes and Surface Area.

Science 2005, 309 (5743), 2040-2042.

52. Jiang, H.-L.; Feng, D.; Liu, T.-F.; Li, J.-R.; Zhou, H.-C., Pore Surface Engineering with Controlled Loadings of Functional Groups via Click Chemistry in Highly Stable Metal-Organic Frameworks. Journal of the American Chemical Society 2012, 134 (36), 14690-14693.

53. Mellot-Draznieks, C.; Dutour, J.; Férey, G., Computational Design of Hybrid Frameworks: Structure and Energetics of Two Me3OF3\{-O2C-C6H4-CO2-\}3 Metal-Dicarboxylate Polymorphs, MILhypo-1 and MIL-hypo-2. Zeitschrift für anorganische und allgemeine Chemie 2004, 630 (15), 25992604.

54. Wilmer, C. E.; Farha, O. K.; Yildirim, T.; Eryazici, I.; Krungleviciute, V.; Sarjeant, A. A.; Snurr, R. Q.; Hupp, J. T., Gram-scale, high-yield synthesis of a robust metal-organic framework for storing methane and other gases. Energy \& Environmental Science 2013, 6 (4), 1158-1163.

55. Tian, Y.-Q.; Yao, S.-Y.; Gu, D.; Cui, K.-H.; Guo, D.-W.; Zhang, G.; Chen, Z.-X.; Zhao, D.-Y., Cadmium Imidazolate Frameworks with Polymorphism, High Thermal Stability, and a Large Surface Area. Chemistry - A European Journal 2010, 16 (4), 1137-1141.

56. Opletal, G.; Petersen, T. C.; Russo, S. P.; Barnard, A. S., PorosityPlus: characterisation of defective, nanoporous and amorphous materials. Journal of Physics: Materials 2018, 1 (1), 016002.

57. Sarkisov, L., Calculation and visualization of free energy barriers for several VOCs and TNT in HKUST-1. Physical Chemistry Chemical Physics 2012, 14 (44), 15438-15444.

58. Sarkisov, L., Toward Rational Design of Metal-Organic Frameworks for Sensing Applications: Efficient Calculation of Adsorption Characteristics in Zero Loading Regime. The Journal of Physical Chemistry C 2012, 116 (4), 3025-3033.

59. Mace, A.; Barthel, S.; Smit, B., Automated Multiscale Approach To Predict Self-Diffusion from a Potential Energy Field. Journal of Chemical Theory and Computation 2019, 15 (4), 2127-2141. 60. Moosavi, S. M.; Nandy, A.; Jablonka, K. M.; Ongari, D.; Janet, J. P.; Boyd, P. G.; Lee, Y.; Smit, B.; Kulik, H. J., Understanding the diversity of the metal-organic framework ecosystem. Nature Communications 2020, 11 (1), 4068. 